Supporting Information for:

\title{
Hybrid Nafion Membranes of Ionic Hydrogen-Bonded Organic Framework Materials for Proton Conduction and PEMFC Applications
}

Xiao-Qian Xu, ${ }^{\dagger}$ Li-Hui Cao, ${ }^{\dagger}, *$ Yan Yang, ${ }^{\dagger}$ Fang Zhao, ${ }^{\dagger}$ Xiang-Tian Bai, ${ }^{\dagger}$ and ShuangQuan Zang*

${ }^{\dagger}$ Shaanxi Key Laboratory of Chemical Additives for Industry, College of Chemistry and Chemical Engineering, Shaanxi University of Science and Technology, Xi'an, 710021, China

*Henan Key Laboratory of Crystalline Molecular Functional Materials, College of Chemistry, Zhengzhou University, Zhengzhou, 450001, China

*E-mail: caolihui @ sust.edu.cn 


\begin{tabular}{|c|c|}
\hline \multicolumn{2}{|l|}{ Table of Contents } \\
\hline Section S1. Experimental details. & Page S3 \\
\hline Section S2. Single crystal X-ray diffraction analyses. & Page S7 \\
\hline Section S3. ${ }^{1} H$ NMR spectra. & Page S13 \\
\hline Section S4. IR spectra. & Page S15 \\
\hline Section S5. Powder X-ray diffraction. & Page S16 \\
\hline Section S6. Scanning Electron Microscope. & Page S20 \\
\hline Section S7. Atomic force microscopy. & Page S21 \\
\hline Section S8. Thermogravimetric analysis. & Page S22 \\
\hline Section S9. Proton conductivity. & Page S24 \\
\hline Section S10. Stress-strain curves. & Page S30 \\
\hline Section S11. References. & Page S31 \\
\hline
\end{tabular}


Section S1. Experimental details.

Materials. 1,5-naphthylene disulfonic acid, Disodium 2,6-Naphthalenedisulfonate, Disodium 2,7-Naphthalenedisulfonate, Disodium 1,6-Naphthalenedisulfonate and Nafion solution $(20 \mathrm{wt} \%)$ were obtained from commercial vendors. DBpy-2I was synthesized according to the published report ${ }^{[\mathrm{S} 1]}$. The solvents used in the experiments were obtained commercially, unless otherwise specified, all starting solvents and materials were used without further purification.

Instrumentation. Powder X-ray diffraction (PXRD) measurements were carried out in Bruker D8 Advance with a $\mathrm{Cu}$ X-ray source over a range of $2 \theta=5.0 \sim 50.0^{\circ}$. Fouriertransform infrared (FT-IR) spectra were obtained on a Bruker VECTOR-22 FTIR spectrometer in the $4000 \sim 400 \mathrm{~cm}^{-1}$ region with $\mathrm{KBr}$ pellets. ${ }^{1} \mathrm{H}$ NMR spectra were performed on a Bruker-600 MHz NMR spectrometer. Chemical shifts are reported in ppm using tetramethylsilane (TMS) as the internal standard. Thermogravimetric analysis (TGA) measurements were performed on a TGA-55 instrument from room temperature to $800{ }^{\circ} \mathrm{C}$ at a heating rate of $10{ }^{\circ} \mathrm{C} / \mathrm{min}$ under air atmosphere. Scanning electron microscope test were performed on a SU8100. The mechanical properties of the membrane are tested on CMT4202 for tensile tester.

Stability experiments. The crystals of iHOFs 1-4 were soaked in water for one week as well as refluxed in boiling water for 24 hours for hydrothermal stability, after the water-treated, the solids were filtered out and dried at room temperature for PXRD measurements. The chemical stability of iHOFs 1-4 were test as follows: the crystal of 
samples was dispersed in $\mathrm{HCl}$ or $\mathrm{NaOH}$ aqueous solutions with different $\mathrm{pH}$ values for 24 hours $(\mathrm{pH}=1,9)$, respectively. After that, the solids were filtered out and dried at room temperature for PXRD to determine its stability. We also performed a PXRD test on the crystal to determine the stability after the electrochemical impedance test.

Proton exchange membrane performance test. The fuel cell performance of composite membrane was evaluated by analyzing the curves of current density versus voltage and power density. The polarization curve is measured by multi-step constant current method. The composite membrane (active area: $5 \mathrm{~cm}^{2}$ ) was sandwichedbetween the two electrodes and then tested in a fuel cell device at $80{ }^{\circ} \mathrm{C}$ under $150 \mathrm{sccm}$ flow rate of $\mathrm{H}_{2}$ and $600 \mathrm{sccm}$ of air with $0.2 \mathrm{MPa}$ back-pressure and $100 \% \mathrm{RH}$ gas humidification, the anode catalyst loading was $0.20 \mathrm{mg} \cdot \mathrm{cm}^{-2}$, and the cathode was 0.4 $\mathrm{mg} \cdot \mathrm{cm}^{-2}$. For the durability analysis, the prepared MEA were first assembled in a single cell conditioned for $4 \mathrm{~h}$ prior to the polarization curve recording. The accelerated life time (ALT) test was conducted through a step sweeping mode (chronoamperometric) at $0.6 \mathrm{~V}$. The test conditions were $80^{\circ} \mathrm{C}$ and $100 \% \mathrm{RH}$; the current density was measured for $20 \mathrm{~h}$.

Preparation of the MEA. The MEA was constructed by directly coated the catalyst slurry on proton exchange membrane to prepare Catalytic layer and then combine with GDL. The catalyst slurry is prepared by fully and evenly mixing platinum-carbon catalyst (Johnmn Matthey, 60\%), 5\% Nafion solution and isopropanol. Coating the prepared catalyst slurry on the upper and lower surfaces of proton exchange membrane, 
and then bonding the membrane with GDL layer (Toray TGP-H-060, 20wt\% PTFE)

coated with conductive adhesive to obtain the membrane electrode.

Proton conductivity. Electrochemical impedance test of the crystal samples was determined by sandwiching the pellets of $\mathrm{iHOFs}$ between two copper sheets and then by two-electrode AC impedance spectroscopy using an electrochemical work-station $(\mathrm{CHI} 660 \mathrm{E})$ in the frequency range from $1 \mathrm{~Hz}$ to $0.1 \mathrm{MHz}$ with an alternating voltage of $5 \mathrm{mV}$.

The samples were pressed into a circular cylinder with a diameter of about $13 \mathrm{~mm}$ on a tableting machine for $5 \mathrm{~min}$ under $10.00 \mathrm{MPa}$ pressure. Their thicknesses and diameter were determined by a Vernier caliper. The temperature and relative humidity conditions are in the range of $30-100{ }^{\circ} \mathrm{C}$ at $75 \% \mathrm{RH}$ to $98 \% \mathrm{RH}$ and the humidity are controlled using high and low temperature and humidity test chamber. The proton conductivity of crystal samples was obtained from the following equation: $\sigma=\mathrm{L} / \mathrm{RS}$, where $\sigma$ is the value of proton conductivity $\left(\mathrm{S} \cdot \mathrm{cm}^{-1}\right), \mathrm{L}$ is the thickness $(\mathrm{cm})$ of the pellet, $\mathrm{R}$ is the value of electrochemical impedance and $\mathrm{S}$ is the flat surface area $\left(\mathrm{cm}^{2}\right)$ calculated by the diameter of the circular cylinder. Activation energy $\left(E_{\mathrm{a}}\right)$ values were calculated from the Arrhenius equation: $\mathrm{T} \sigma=\sigma_{0} \exp \left(-E_{\mathrm{a}} / \mathrm{kT}\right)$, where $\sigma_{0}$ is the pre-exponential factor, $\mathrm{T}$ is temperature, $\mathrm{k}$ is Boltzmann constant.

Water uptake and area swelling. Water uptake and area swelling test were measured to investigate the dimensional stability and hydrophilic ability of membrane. The weights $\left(W_{\text {dry }}, \mathrm{g}\right)$ and area $\left(A_{\mathrm{dry}}, \mathrm{cm}^{2}\right)$ were pre-measured before testing. The area was 
measured by the length of the composite membrane. The composite membrane was cut into pieces $\left(1 \times 1 \mathrm{~cm}^{2}\right)$ and then immersed into deionized water for $48 \mathrm{~h}$ at room temperature. After that, the weights $\left(W_{\text {wet }}, \mathrm{g}\right)$ and areas $\left(A_{\text {wet }}, \mathrm{cm}^{2}\right)$ of the membrane were calculated immediately after wiping off the moisture on the surface. The water uptake and swelling rates were calculated using the following equations:

water uptake $=\left[\left(W_{\text {wet }}-W_{\text {dry }}\right) / W_{\text {dry }}\right] \times 100 \%$

area swelling $=\left[\left(A_{\text {wet }}-A_{\text {dry }}\right) / A_{\text {dry }}\right] \times 100 \%$

Gas permeation. The air permeability of the membrane is tested by differential pressure method. The time required to measure the penetration of the same volume of gas through the membrane.

The calculation formula is: Gas degree $=1.27 \mathrm{Vt}(\mathrm{um} / \mathrm{Pa} \cdot \mathrm{s})$

$\mathrm{V}$ : The amount of air passing through the sample;

$\mathrm{t}$ : Time for the sample to pass through $\mathrm{V} \mathrm{ml}$ air. 


\section{Section S2: Single crystal X-ray diffraction analyses.}

Single-crystal X-ray diffraction data for compounds iHOFs 1-4 were collected on a Bruker SMART APEX CCD diffractometer ${ }^{[\mathrm{S} 2]}$ equipped with a graphitemonochromated Mo-K $\alpha$ radiation $(\lambda=0.71073 \AA)$ using the $\omega$-scan technique. Data reduction was performed using SAINT and corrected for Lorentz and polarization effects. Adsorption corrections were applied using the SADABS routine. ${ }^{[\mathrm{S} 3]}$ All the structures were solved with direct methods $(S H E L X S)^{[\mathrm{S} 4]}$ and refined by full-matrix least squares on $F^{2}$ using $O L E X 2,{ }^{[\mathrm{S} 5]}$ which utilizes the $S H E L X L-2015$ module. ${ }^{[\mathrm{S} 6]}$ All non-hydrogen atoms were refined anisotropically. Displacement parameter restraints were used in modeling the ligands. Hydrogen atoms were placed geometrically on their riding atom where possible. The contents of the solvent region are not represented in the unit cell contents in the crystal data. Crystal data containing space group, lattice parameters and other relevant information for the title complex are summarized in Table S1, and hydrogen bonds data details are summarized in Table S2. More details on the crystallographic data are given in the X-ray crystallographic files in CIF format. Full details of the structure determinations have been deposited with Cambridge Crystallographic Data Center under reference number CCDC 2069880 2069883 for iHOFs 1-4, and are available free of charge from CCDC. 
Table S1. Crystal structure data and refinement details of iHOFs 1-4.

\begin{tabular}{|c|c|c|c|c|}
\hline Compounds & iHOF-1 & iHOF-2 & iHOF-3 & iHOF-4 \\
\hline Empirical formula & $\mathrm{C}_{20} \mathrm{H}_{18} \mathrm{~N}_{4} \mathrm{O}_{6} \mathrm{~S}_{2}$ & $\mathrm{C}_{20} \mathrm{H}_{22} \mathrm{~N}_{4} \mathrm{O}_{8} \mathrm{~S}_{2}$ & $\mathrm{C}_{20} \mathrm{H}_{22} \mathrm{~N}_{4} \mathrm{O}_{8} \mathrm{~S}_{2}$ & $\mathrm{C}_{20} \mathrm{H}_{22} \mathrm{~N}_{4} \mathrm{O}_{8} \mathrm{~S}_{2}$ \\
\hline Formula weight & 474.50 & 510.53 & 510.53 & 510.53 \\
\hline Temperature / K & 273.15 & 296.15 & 296.15 & 296.15 \\
\hline Wavelength / $\AA$ & 0.71073 & 0.71073 & 0.71073 & 0.71073 \\
\hline Crystal system & monoclinic & orthorhombic & monoclinic & monoclinic \\
\hline Space group & $P 2_{1} / n$ & $P b c a$ & $P 2_{1} / n$ & $P 2_{1} / n$ \\
\hline$a / \AA$ & $7.1525(9)$ & $15.2513(8)$ & $7.144(3)$ & $7.552(4)$ \\
\hline$b / \AA ̊ ̊$ & $19.669(2)$ & $13.8071(8)$ & $18.382(7)$ & $14.641(8)$ \\
\hline$c / \AA ̊$ & $14.3443(16)$ & $20.4803(12)$ & $8.540(3)$ & $9.837(5)$ \\
\hline$\alpha /^{\circ}$ & 90.00 & 90 & 90 & 90 \\
\hline$\beta 1^{\circ}$ & $90.578(7)$ & 90 & $106.523(5)$ & $90.740(9)$ \\
\hline$\gamma /{ }^{\circ}$ & 90.00 & 90 & 90 & 90 \\
\hline Volume $/ \AA^{3}$ & $2017.9(4)$ & $4312.7(4)$ & $1075.2(7)$ & $1087.6(10)$ \\
\hline $\mathrm{Z}$ & 4 & 8 & 2 & 2 \\
\hline Density (calculated) $/ \mathrm{g} \cdot \mathrm{cm}^{-3}$ & 1.562 & 1.573 & 1.577 & 1.559 \\
\hline Absorption coefficient $/ \mathrm{mm}^{-1}$ & 0.313 & 0.305 & 0.306 & 0.303 \\
\hline $\mathrm{F}(000)$ & 984.0 & 2128.0 & 532.0 & 532.0 \\
\hline Reflections collected & 17406 & 38552 & 9037 & 11521 \\
\hline \multirow[t]{2}{*}{ Independent reflections } & 3543 & 4944 & 1526 & 1909 \\
\hline & $\left(\mathrm{R}_{\mathrm{int}}=0.0282\right)$ & $\left(\mathrm{R}_{\mathrm{int}}=0.0973\right)$ & $\left(\mathrm{R}_{\mathrm{int}}=0.0404\right)$ & $\left(\mathrm{R}_{\mathrm{int}}=0.0338\right)$ \\
\hline Data/restraints/parameters & $3543 / 0 / 295$ & $4944 / 0 / 315$ & $1526 / 0 / 158$ & $1909 / 0 / 158$ \\
\hline Goodness-of-fit on $F^{2}$ & 1.061 & 1.058 & 1.057 & 1.100 \\
\hline${ }^{a} R_{1},{ }^{b} w R_{2}[I>2 \sigma(I)]$ & $0.0425 / 0.1060$ & $0.0519 / 0.1162$ & $0.0532 / 0.1469$ & $0.0323 / 0.0931$ \\
\hline${ }^{a} R_{1},{ }^{b} w R_{2}$ (all data) & $0.0485 / 0.1089$ & $0.0885 / 0.1354$ & $0.0636 / 0.1539$ & $0.0396 / 0.0976$ \\
\hline Largest diff. peak and hole & $0.34 /-0.29$ e. $\AA^{-3}$ & $0.63 /-0.69$ e. $\AA^{-3}$ & $0.89 /-0.35$ e. $\AA^{-3}$ & $0.31 /-0.25$ e. $\AA^{-3}$ \\
\hline CCDC number & 2069880 & 2069881 & 2069882 & 2069883 \\
\hline
\end{tabular}$$
{ }^{a} R_{1}=\sum|| F_{\mathrm{o}}|-| F_{\mathrm{c}}|| / \sum\left|F_{\mathrm{o}}\right| .{ }^{b} w R_{2}=\left\{\sum\left[w\left(F_{\mathrm{o}}{ }^{2}-F_{\mathrm{c}}{ }^{2}\right)^{2}\right] / \sum\left[w\left(F_{\mathrm{o}}{ }^{2}\right)^{2}\right]\right\}^{1 / 2}
$$ 
(a)

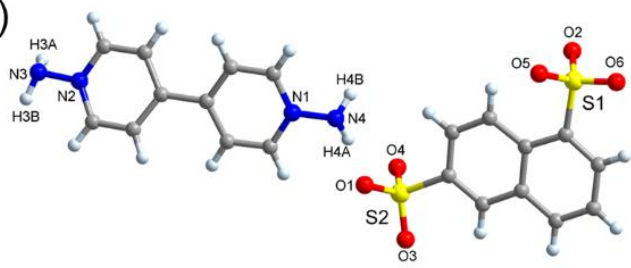

(b)

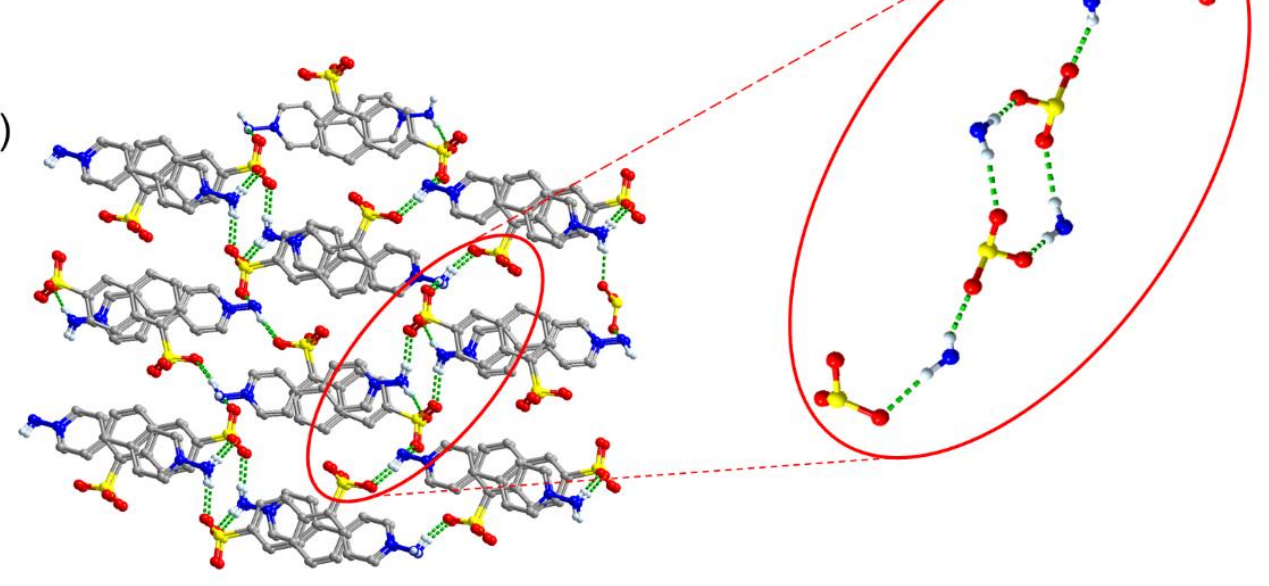

Figure S1. (a) The asymmetric unit of iHOF-1. (b) The supramolecular structure of iHOF-1. (c) The 0D hydrogen bonds in the structure of iHOF-1.

(a)

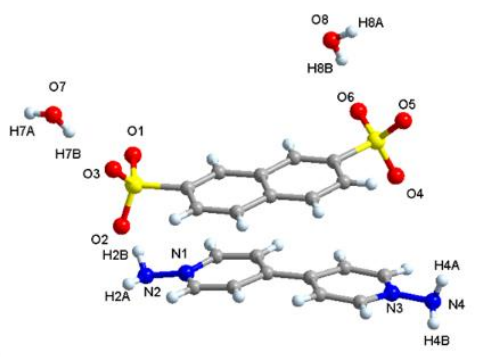

(c)

(b)
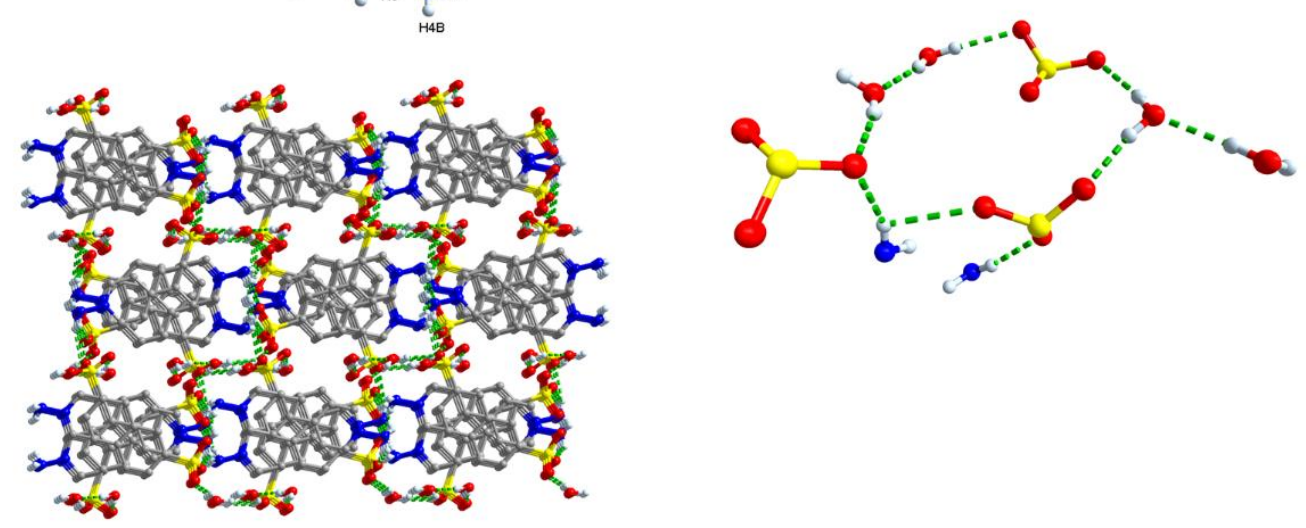

Figure S2. (a) The asymmetric unit of iHOF-2. (b) The supramolecular structure of iHOF-2. (c) The hydrogen bond in the structure of iHOF-2. 

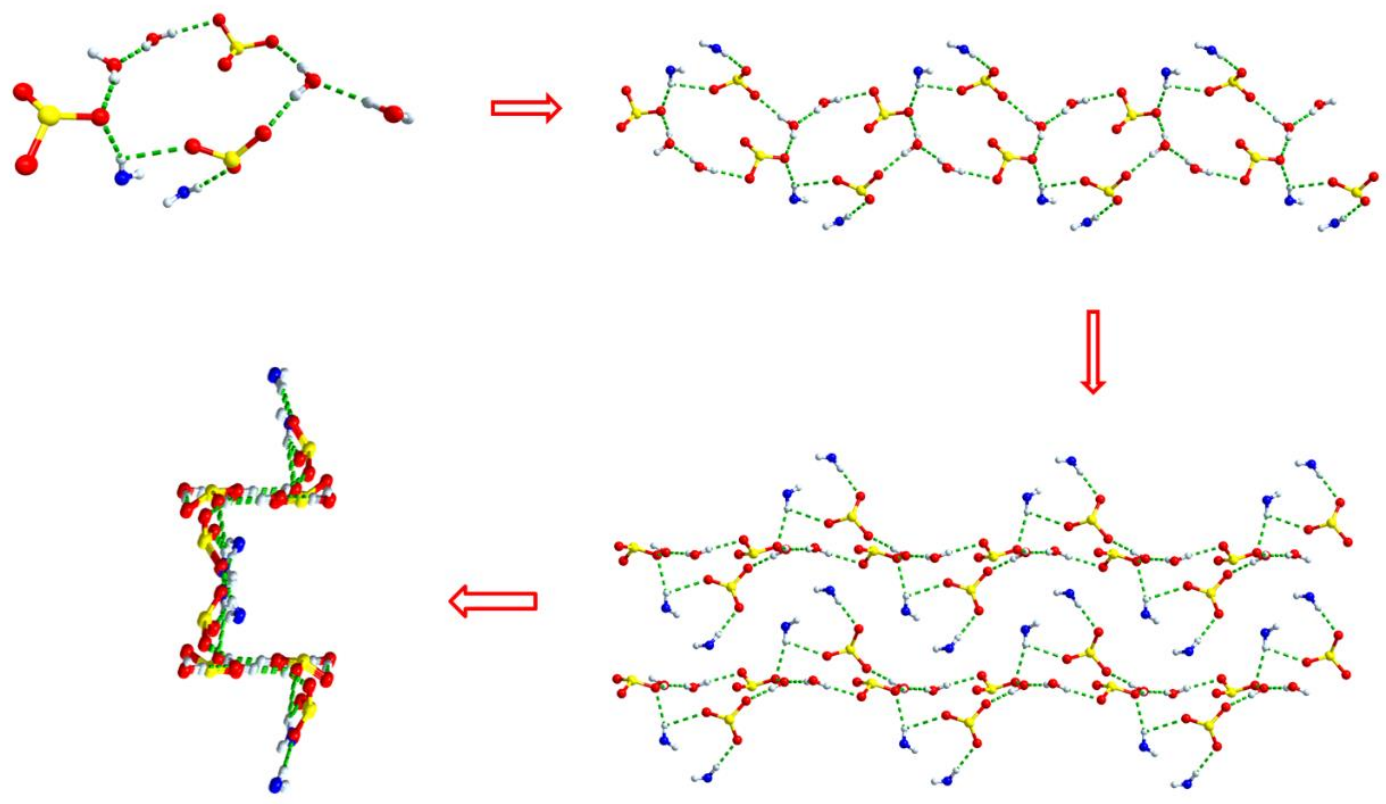

Figure S3. The 1D hydrogen bond in the structure of iHOF-2.

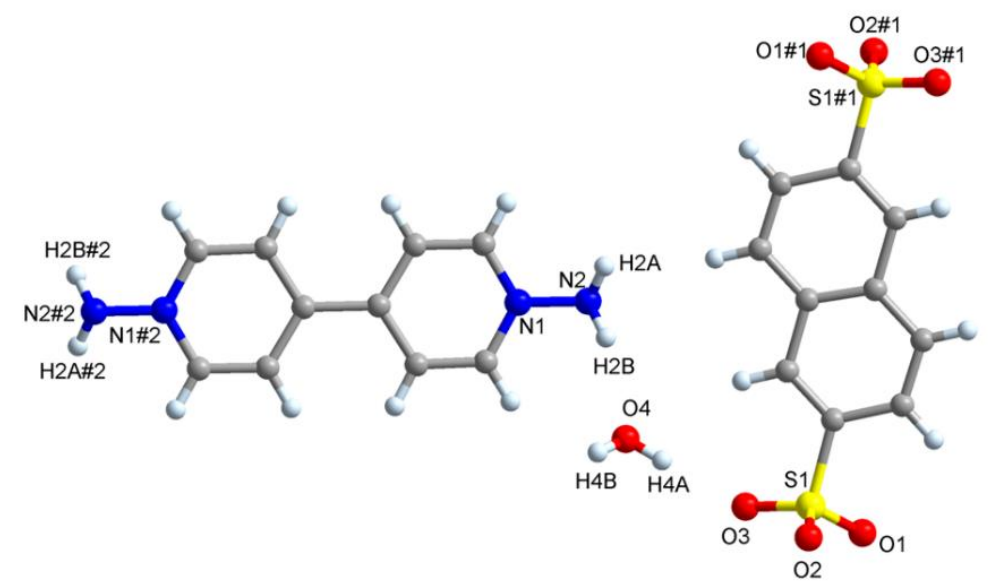

Figure S4. The asymmetric unit of iHOF-3. 
(a)

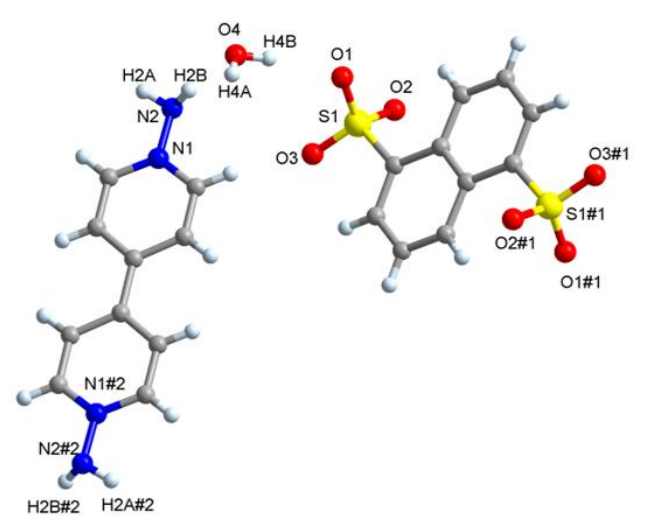

(b)
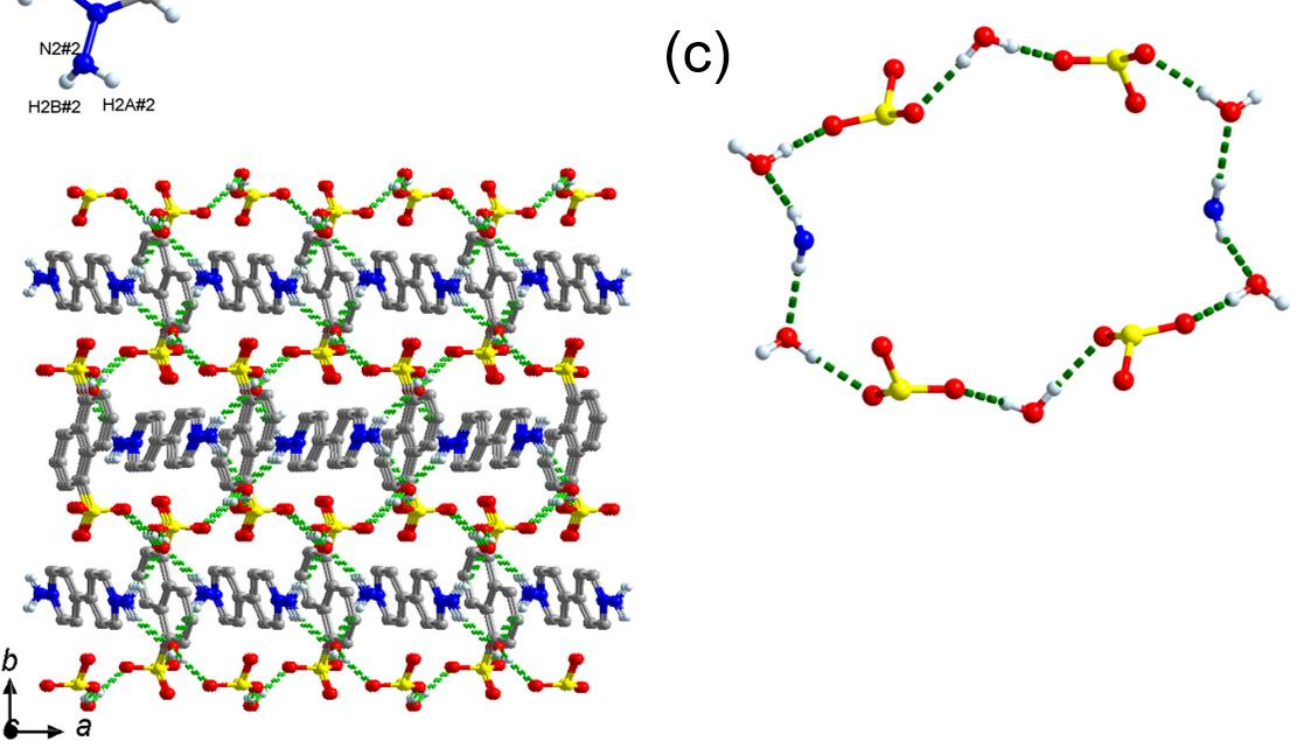

Figure S5. (a) The structure unit of iHOF-4. (b) Stacking in an $A B A B$ fashion of the iHOF-4 supermolecular structure. (c) The hydrogen bonding unit of 32 membered ring. 
Table S2. Hydrogen Bonds data details of iHOFs 1-4.

\begin{tabular}{|c|c|c|c|c|c|c|c|c|}
\hline \multicolumn{9}{|c|}{ iHOF-1 } \\
\hline $\mathrm{X} \cdots \mathrm{Y} / \AA$ & $\mathrm{N} 4 \cdots \mathrm{O} 1$ & 2.877 & $\mathrm{~N} 4 \cdots \mathrm{O} 4$ & 2.932 & $\mathrm{~N} 3 \cdots \mathrm{O} 3$ & 2.973 & $\mathrm{~N} 3 \cdots \mathrm{O} 6$ & 2.906 \\
\hline $\mathrm{H} \cdots \mathrm{Y} / \AA$ & $\mathrm{H} 4 \mathrm{~A} \cdots \mathrm{O} 1$ & 2.031 & $\mathrm{H} 4 \mathrm{~B} \cdots \mathrm{O} 4$ & 2.094 & $\mathrm{H} 3 \mathrm{~A} \cdots \mathrm{O} 3$ & 2.158 & $\mathrm{H} 3 \mathrm{~B} \cdots \mathrm{O} 6$ & 2.041 \\
\hline$\angle \mathrm{XHY} /{ }^{\circ}$ & \multicolumn{2}{|c|}{$\angle \mathrm{N} 4 \mathrm{H} 4 \mathrm{AO} 1 \quad 166$} & \multicolumn{2}{|c|}{$\angle \mathrm{N} 4 \mathrm{H} 4 \mathrm{BO} 4 \quad 162$} & \multicolumn{2}{|c|}{$\angle \mathrm{N} 3 \mathrm{H} 3 \mathrm{AO} 3 \quad 158$} & \multicolumn{2}{|c|}{$\angle \mathrm{N} 3 \mathrm{H} 3 \mathrm{BO} 6 \quad 157$} \\
\hline \multicolumn{9}{|c|}{ iHOF-2 } \\
\hline $\mathrm{X} \cdots \mathrm{Y} / \AA$ & $\mathrm{N} 2 \cdots \mathrm{O} 4$ & 3.085 & $\mathrm{~N} 2 \cdots \mathrm{O} 2$ & 2.966 & $\mathrm{~N} 4 \cdots \mathrm{O} 1$ & 2.881 & $\mathrm{O} 7 \cdots \mathrm{O} 4$ & 2.831 \\
\hline $\mathrm{H} \cdots \mathrm{Y} / \AA$ & $\mathrm{H} 2 \mathrm{~A} \cdots \mathrm{O} 4$ & 2.271 & $\mathrm{H} 2 \mathrm{~A} \cdots \mathrm{O} 2$ & 2.584 & $\mathrm{H} 4 \mathrm{~B} \cdots \mathrm{O} 1$ & 2.032 & $\mathrm{H} 7 \mathrm{~A} \cdots \mathrm{O} 4$ & 1.995 \\
\hline$\angle \mathrm{XHY} /{ }^{\circ}$ & \multicolumn{2}{|c|}{$\angle \mathrm{N} 2 \mathrm{H} 2 \mathrm{AO} 4 \quad 157$} & \multicolumn{2}{|c|}{$\angle \mathrm{N} 2 \mathrm{H} 2 \mathrm{AO} 2 \quad 107$} & \multicolumn{2}{|c|}{$\angle \mathrm{N} 4 \mathrm{H} 4 \mathrm{BO} 1 \quad 169$} & \multicolumn{2}{|c|}{$\angle \mathrm{O} 7 \mathrm{H} 7 \mathrm{AO} 4 \quad 167$} \\
\hline $\mathrm{X} \cdots \mathrm{Y} / \AA$ & $\mathrm{O} 7 \cdots \mathrm{O} 3$ & 2.773 & $08 \cdots \mathrm{O} 7$ & 2.792 & $\mathrm{O} 8 \cdots \mathrm{O} 6$ & 2.941 & & \\
\hline $\mathrm{H} \cdots \mathrm{Y} / \AA$ & $\mathrm{H} 7 \mathrm{~B} \cdots \mathrm{O} 3$ & 1.938 & $\mathrm{H} 8 \mathrm{~A} \cdots \mathrm{O} 7$ & 1.967 & $\mathrm{H} 8 \mathrm{~B} \cdots \mathrm{O} 6$ & 2.097 & & \\
\hline$\angle \mathrm{XHY} /{ }^{\circ}$ & \multicolumn{2}{|c|}{$\angle \mathrm{O} 7 \mathrm{H} 7 \mathrm{BO} 3 \quad 166$} & \multicolumn{2}{|c|}{$\angle \mathrm{O} 8 \mathrm{H} 8 \mathrm{AO} 7 \quad 163$} & \multicolumn{2}{|c|}{$\angle$ O8H8BO6 171} & & \\
\hline \multicolumn{9}{|c|}{ iHOF-3 } \\
\hline $\mathrm{X} \cdots \mathrm{Y} / \AA$ & $\mathrm{N} 2 \cdots \mathrm{O} 2$ & 2.965 & $\mathrm{~N} 2 \cdots \mathrm{O} 4$ & 2.948 & $\mathrm{~N} 2 \cdots \mathrm{O} 4$ & 3.462 & $\mathrm{O} 4 \cdots \mathrm{O} 1$ & 3.028 \\
\hline $\mathrm{H} \cdots \mathrm{Y} / \AA$ & $\mathrm{H} 2 \mathrm{~A} \cdots \mathrm{O} 2$ & 2.184 & $\mathrm{H} 2 \mathrm{~B} \cdots \mathrm{O} 4$ & 2.108 & $\mathrm{H} 2 \mathrm{~A} \cdots \mathrm{O} 4$ & 2.859 & $\mathrm{H} 4 \mathrm{~B} \cdots \mathrm{O} 1$ & 2.200 \\
\hline$\angle \mathrm{XHY} 1^{\circ}$ & \multicolumn{2}{|c|}{$\angle \mathrm{N} 2 \mathrm{H} 2 \mathrm{AO} 2 \quad 149$} & \multicolumn{2}{|c|}{$\angle \mathrm{N} 2 \mathrm{H} 2 \mathrm{BO} 4 \quad 163$} & \multicolumn{2}{|c|}{$\angle \mathrm{N} 2 \mathrm{H} 2 \mathrm{AO} 4 \quad 127$} & \multicolumn{2}{|c|}{$\angle \mathrm{O} 4 \mathrm{H} 4 \mathrm{BO} 1 \quad 164$} \\
\hline $\mathrm{X} \cdots \mathrm{Y} / \AA$ & $\mathrm{O} 4 \cdots \mathrm{O} 3$ & 3.098 & $\mathrm{O} 4 \cdots \mathrm{O} 3$ & 2.739 & & & & \\
\hline $\mathrm{H} \cdots \mathrm{Y} / \AA$ & $\mathrm{H} 4 \mathrm{~B} \cdots \mathrm{O} 3$ & 2.622 & $\mathrm{H} 4 \mathrm{~A} \cdots \mathrm{O} 3$ & 1.896 & & & & \\
\hline$\angle \mathrm{XHY} /^{\circ}$ & $\angle \mathrm{O} 4 \mathrm{H} 4 \mathrm{BO} 3$ & $3 \quad 116$ & $\angle \mathrm{O} 4 \mathrm{H} 4 \mathrm{AO}$ & $3 \quad 171$ & & & & \\
\hline & & & & HOF-4 & & & & \\
\hline$X \cdots Y / \AA$ & $\mathrm{N} 2 \cdots \mathrm{O} 4$ & 2.996 & $\mathrm{~N} 2 \cdots \mathrm{O} 4$ & 3.005 & $\mathrm{O} 4 \cdots \mathrm{O} 2$ & 2.743 & $\mathrm{O} 4 \cdots \mathrm{O} 1$ & 2.838 \\
\hline $\mathrm{H} \cdots \mathrm{Y} / \AA$ & $\mathrm{H} 2 \mathrm{~A} \cdots \mathrm{O} 4$ & 2.162 & $\mathrm{H} 2 \mathrm{~B} \cdots \mathrm{O} 4$ & 2.145 & $\mathrm{H} 4 \mathrm{~A} \cdots \mathrm{O} 2$ & 1.912 & $\mathrm{H} 4 \mathrm{~B} \cdots \mathrm{O} 1$ & 1.995 \\
\hline$\angle \mathrm{XHY} /{ }^{\circ}$ & $\angle \mathrm{N} 2 \mathrm{H} 2 \mathrm{AO}$ & $4 \quad 161$ & $\angle \mathrm{N} 2 \mathrm{H} 2 \mathrm{BO}$ & $4 \quad 172$ & $\angle \mathrm{O} 4 \mathrm{H} 4 \mathrm{AO} 2$ & 2165 & $\angle \mathrm{O} 4 \mathrm{H} 4 \mathrm{BO} 1$ & $1 \quad 171$ \\
\hline
\end{tabular}

\section{Section S3. ${ }^{1} \mathrm{H}$ NMR spectra.}




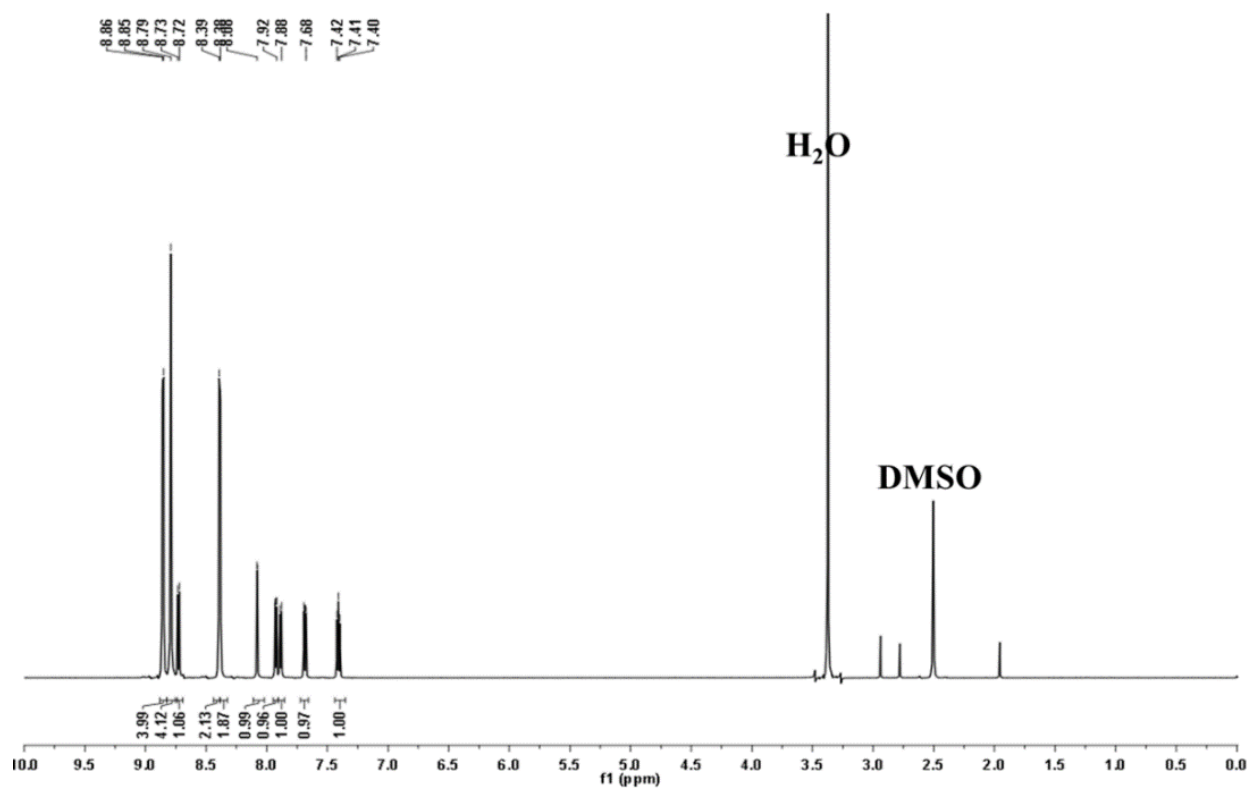

Figure S6. ${ }^{1} \mathrm{H}$ NMR spectrum of iHOF-1 (DMSO-d 6 , $\left.600 \mathrm{MHz}\right)$.

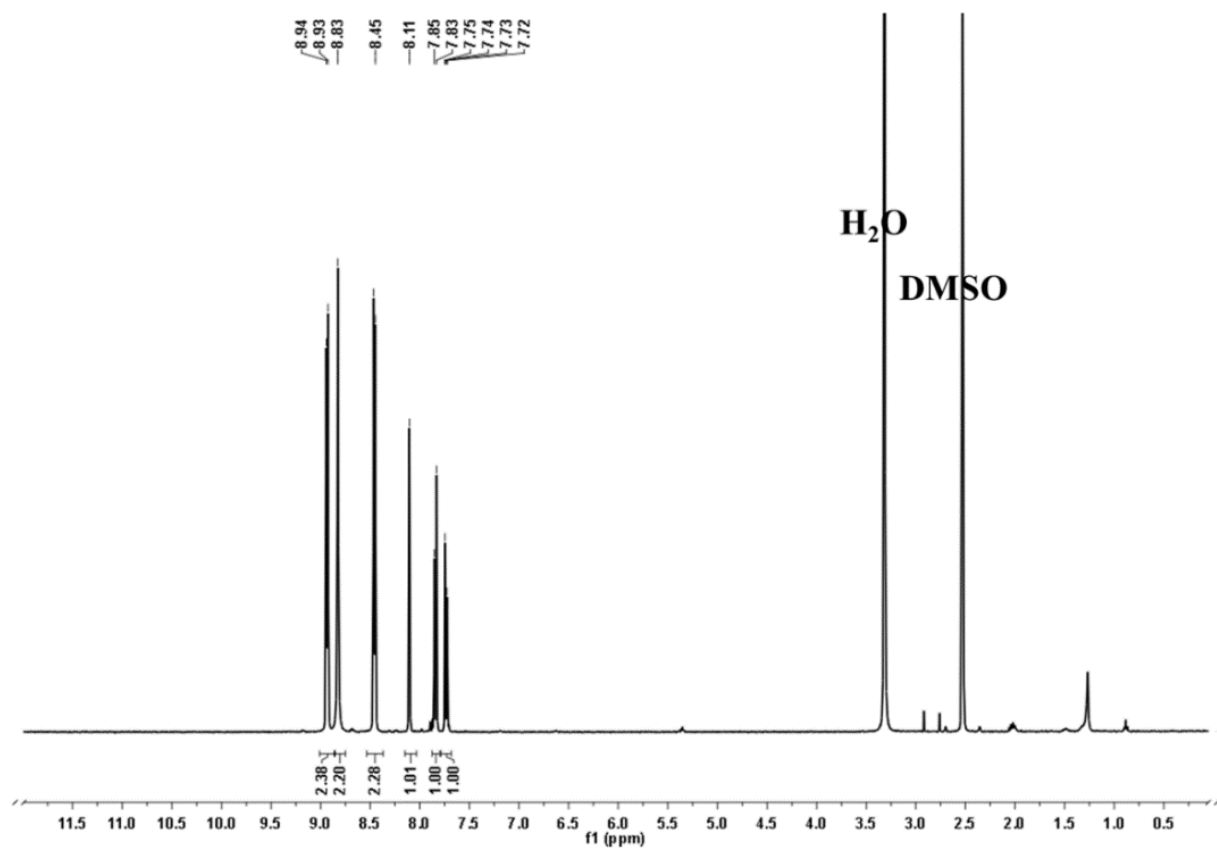

Figure S7. ${ }^{1} \mathrm{H}$ NMR spectrum of iHOF-2 $\left(\mathrm{DMSO}_{-} \mathrm{d}_{6}, 600 \mathrm{MH}_{\mathrm{Z}}\right)$. 


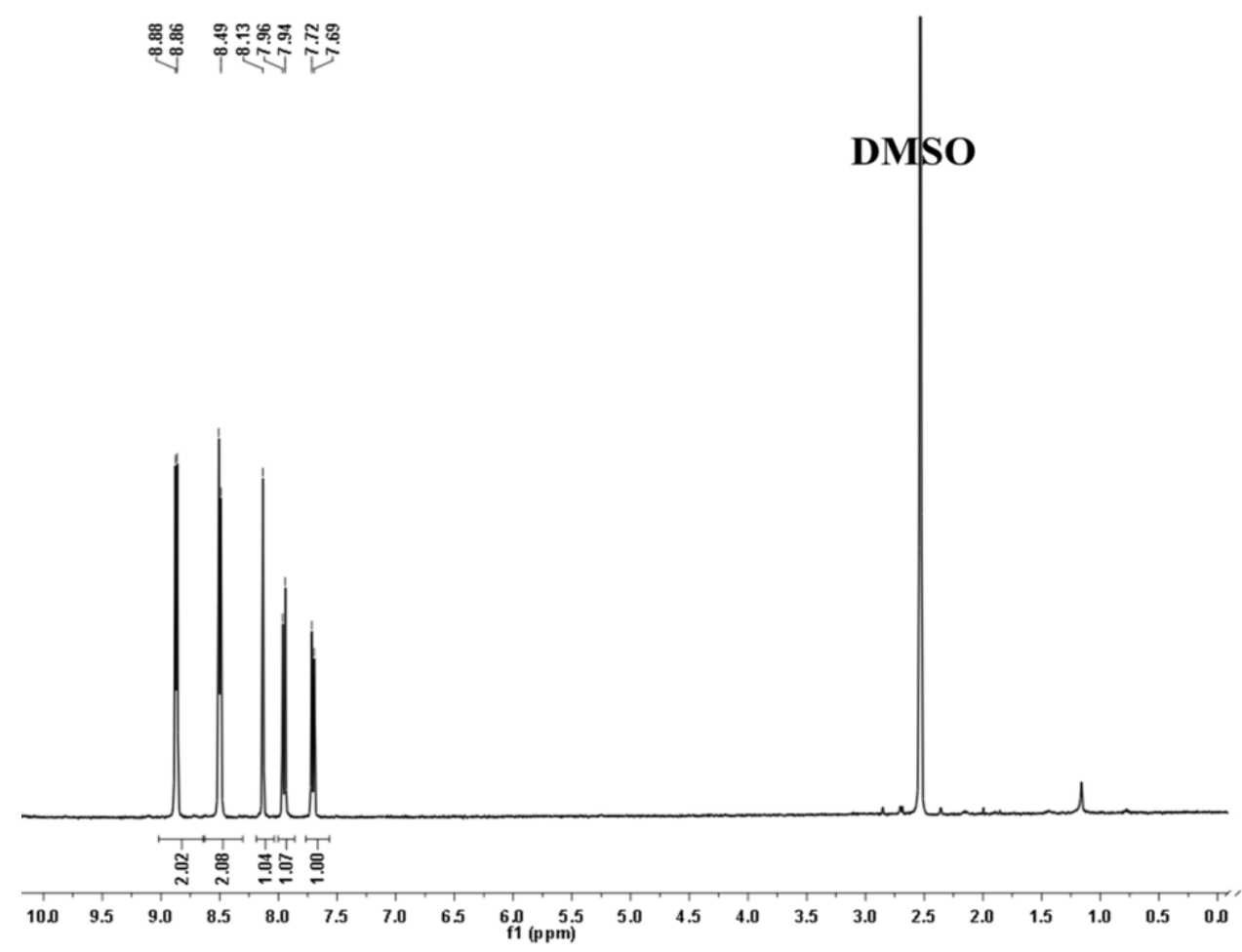

Figure S8. ${ }^{1} \mathrm{H}$ NMR spectrum of iHOF-3 (DMSO-d $\left.6,600 \mathrm{MHz}\right)$.

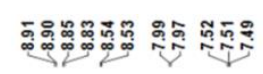

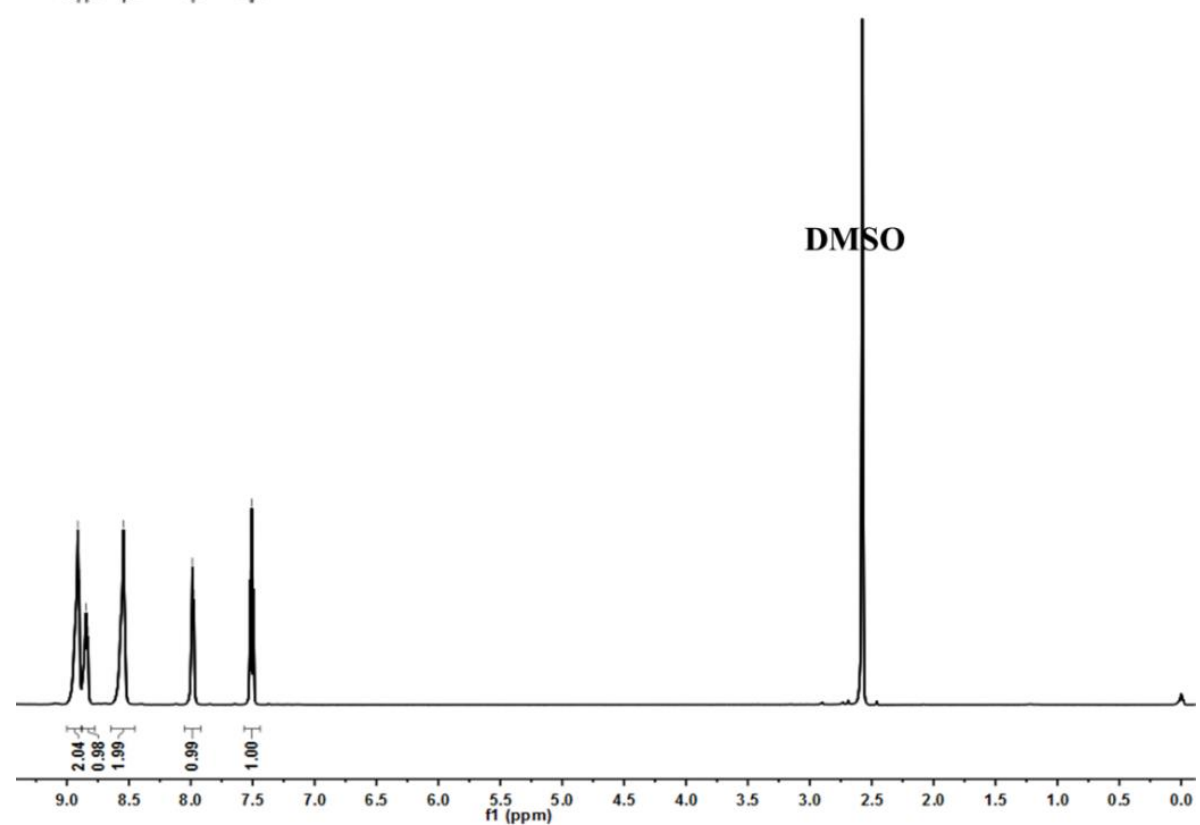

Figure S9. ${ }^{1} \mathrm{H}$ NMR spectrum of iHOF-4 (DMSO-d 6 , $\left.600 \mathrm{MHz}\right)$. 
Section S4. IR spectra.

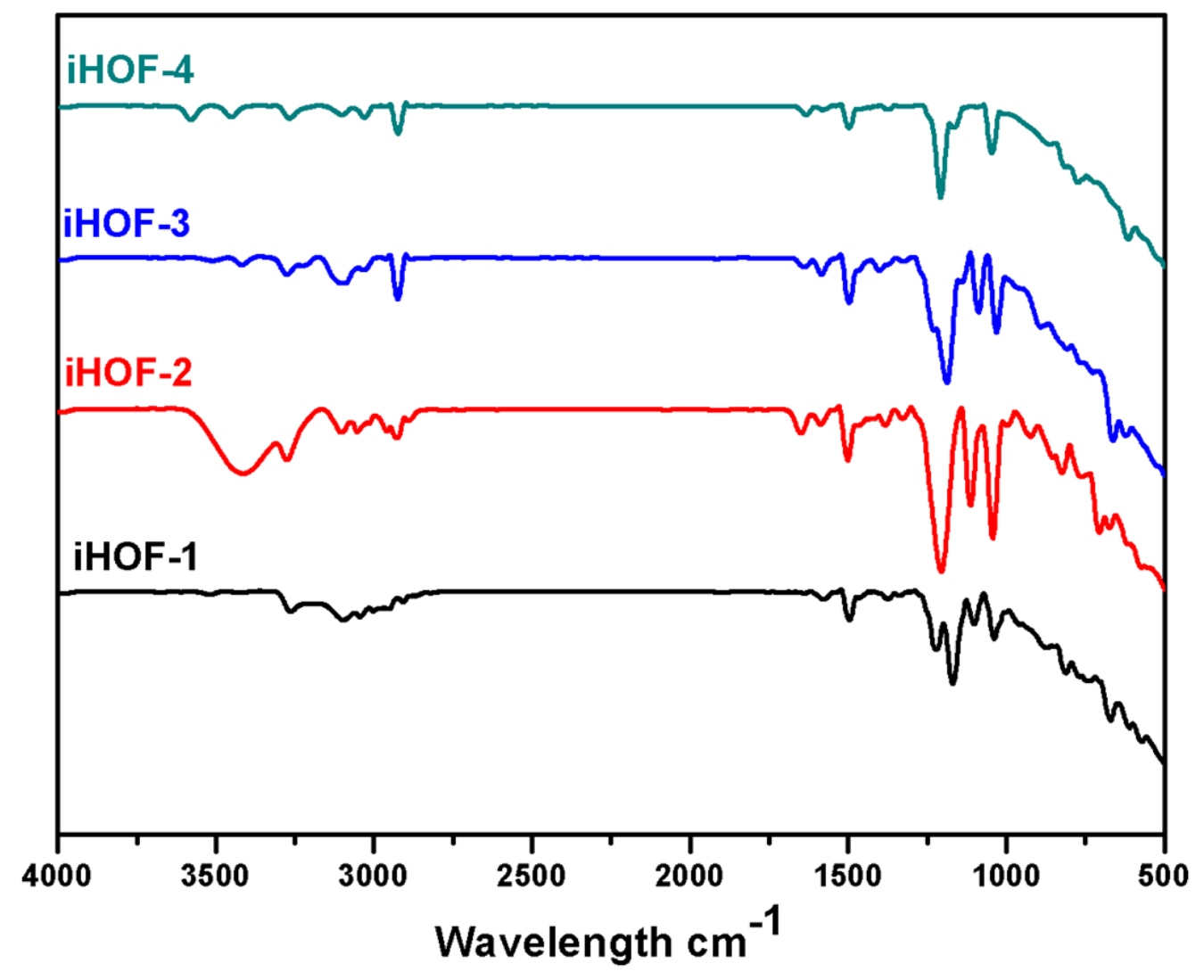

Figure S10. IR spectrum of iHOF 1-4. 
Section S5. Powder X-ray diffraction.

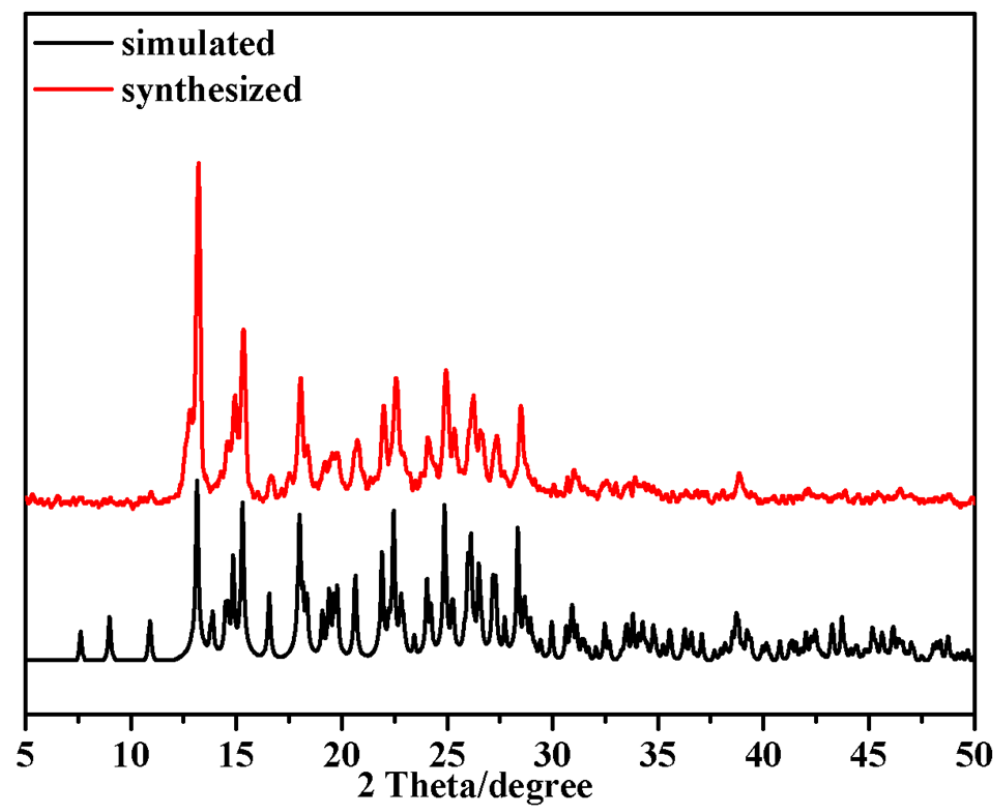

Figure S11. PXRD patterns of iHOF-1 simulated (black) and as-synthesized (red).

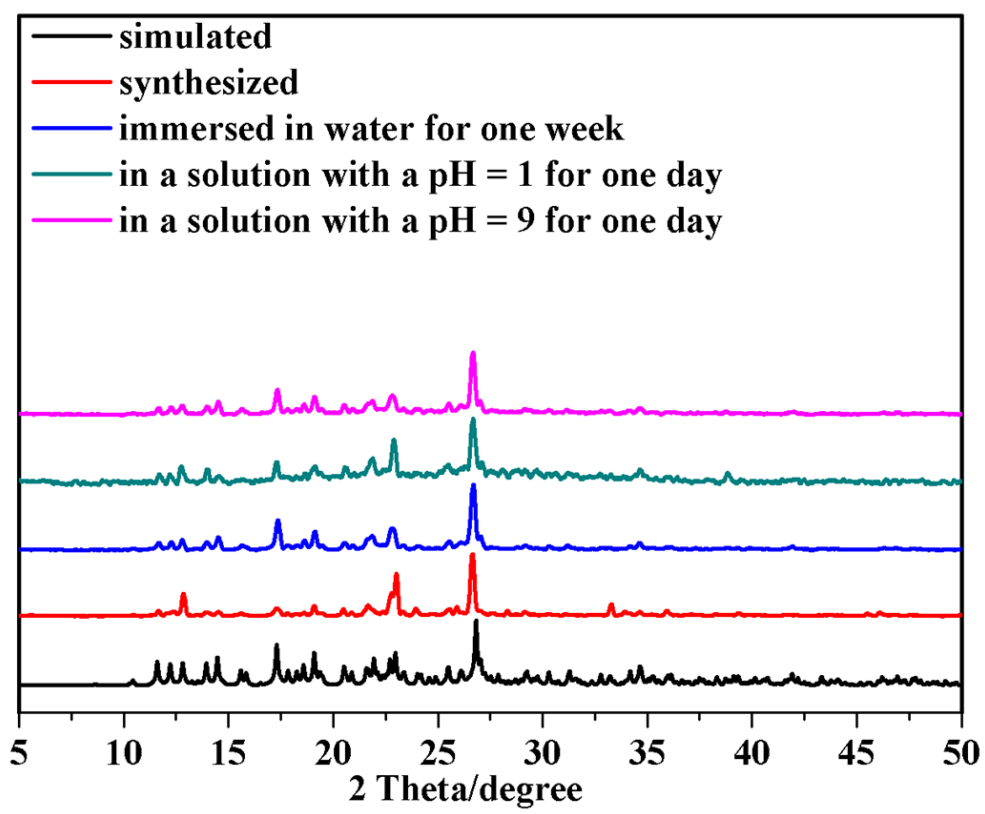

Figure S12. PXRD patterns of iHOF-2 as-synthesized (red), treatment with water for one week (blue), acid solution for one day (green) and Alkaline solution (light red). 

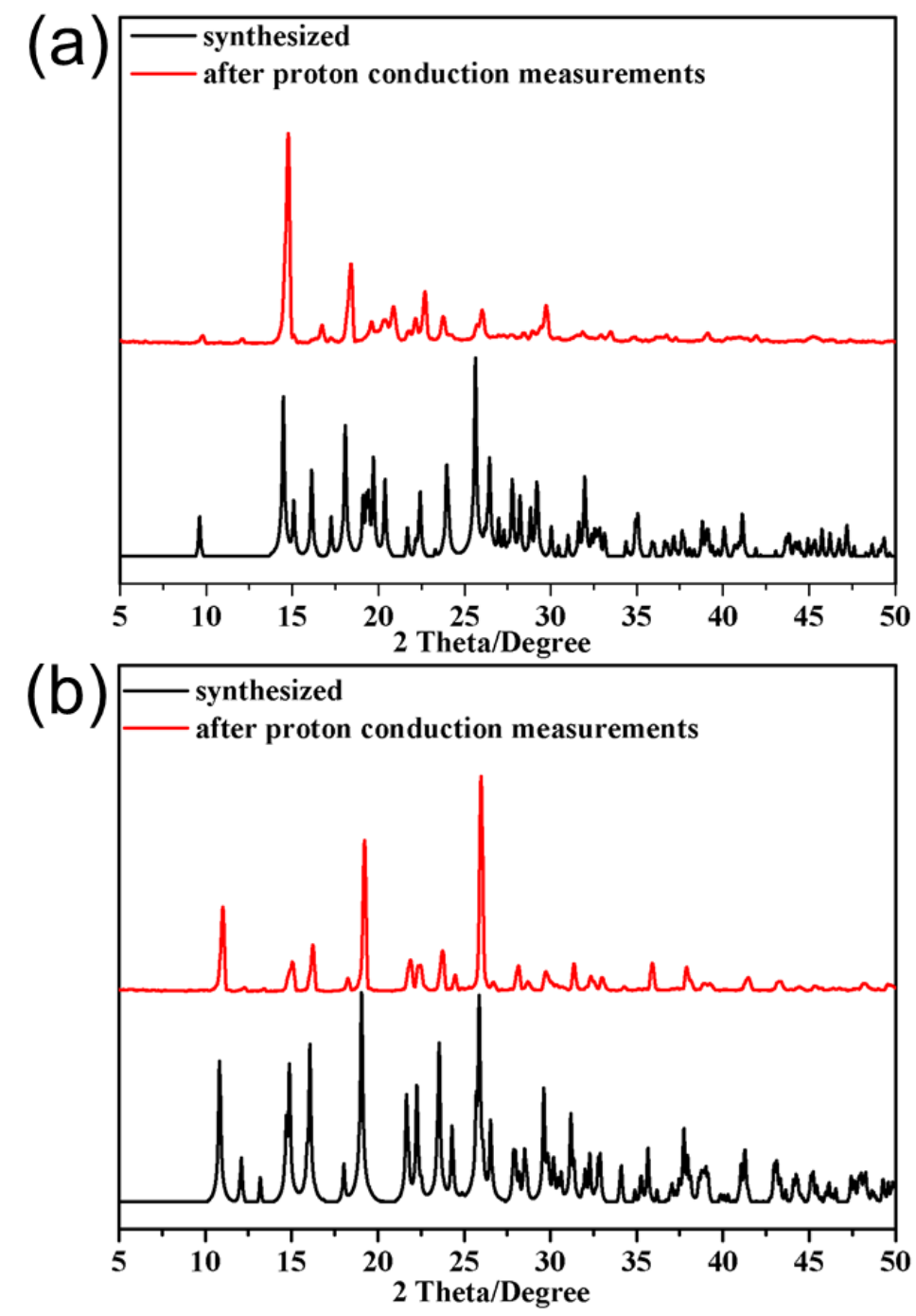

Figure S13. PXRD patterns of iHOF-3 (a) and iHOF-4 (b). Synthesized(black) and after proton conduction measurement(red). 
(a)

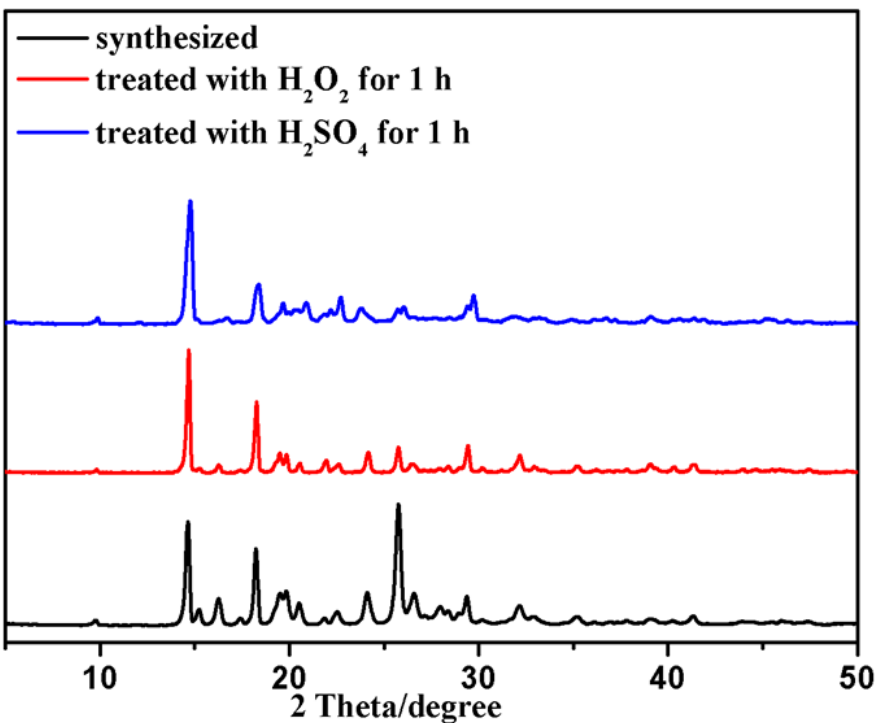

(b)

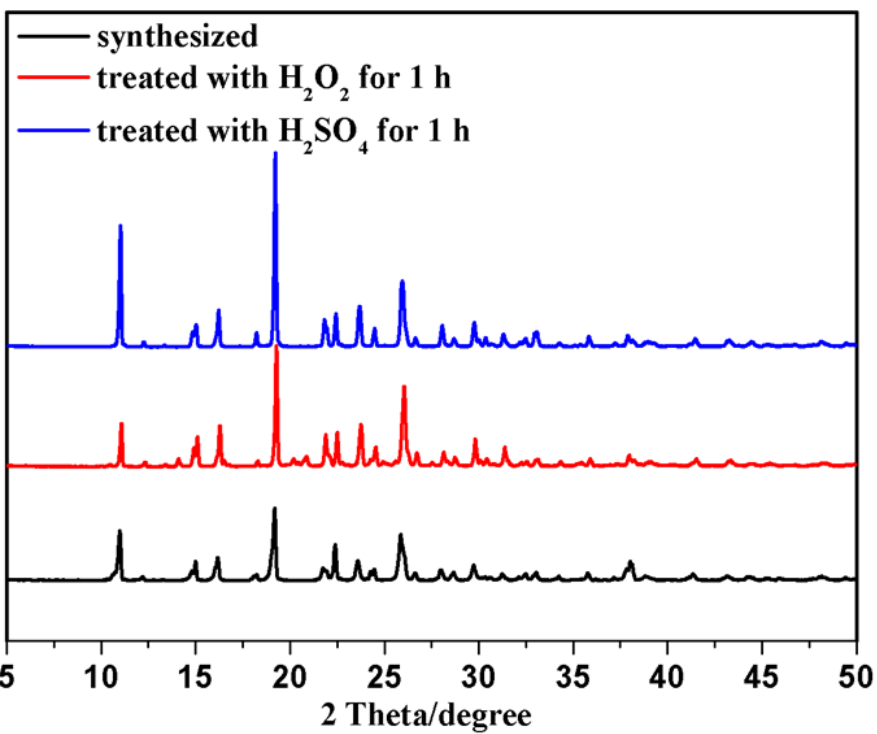

Figure S14. PXRD patterns of iHOF-3 (a) and iHOF-4 (b) treated with $\mathrm{H}_{2} \mathrm{O}_{2}$ and $\mathrm{H}_{2} \mathrm{SO}_{4}$. 


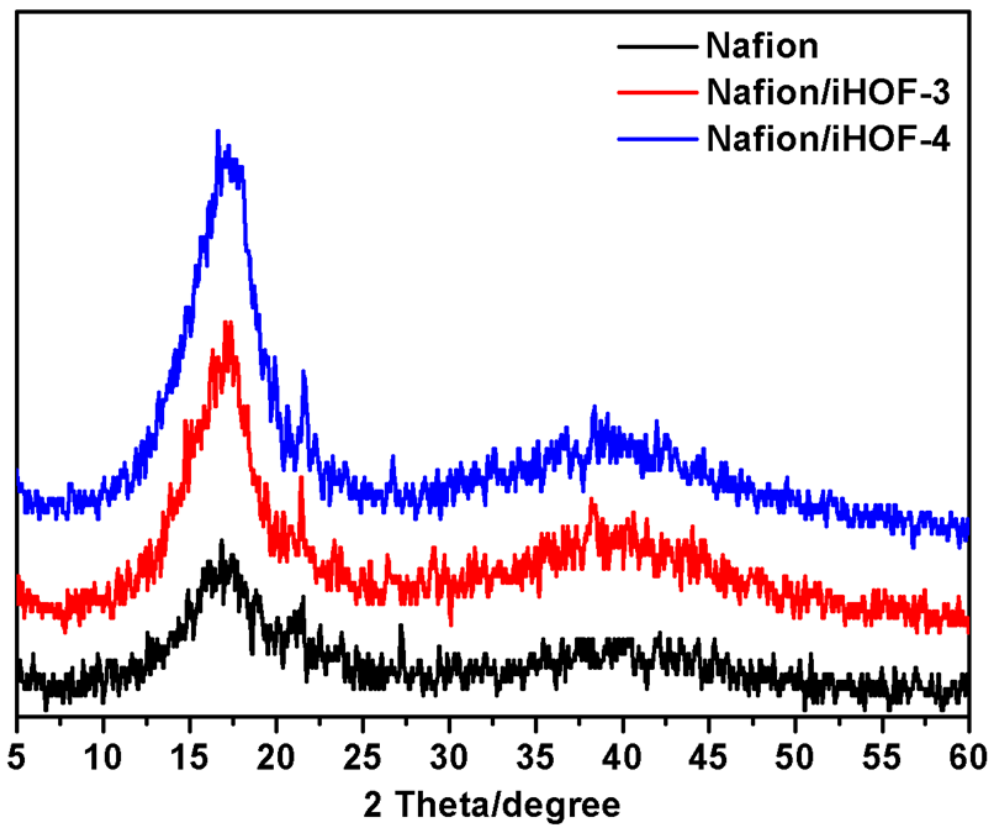

Figure S15. PXRD patterns of iHOF-3 (a) and iHOF-4 (b). Synthesized(black) and after proton conduction measurement(red). 
Section S6. Scanning Electron Microscope.
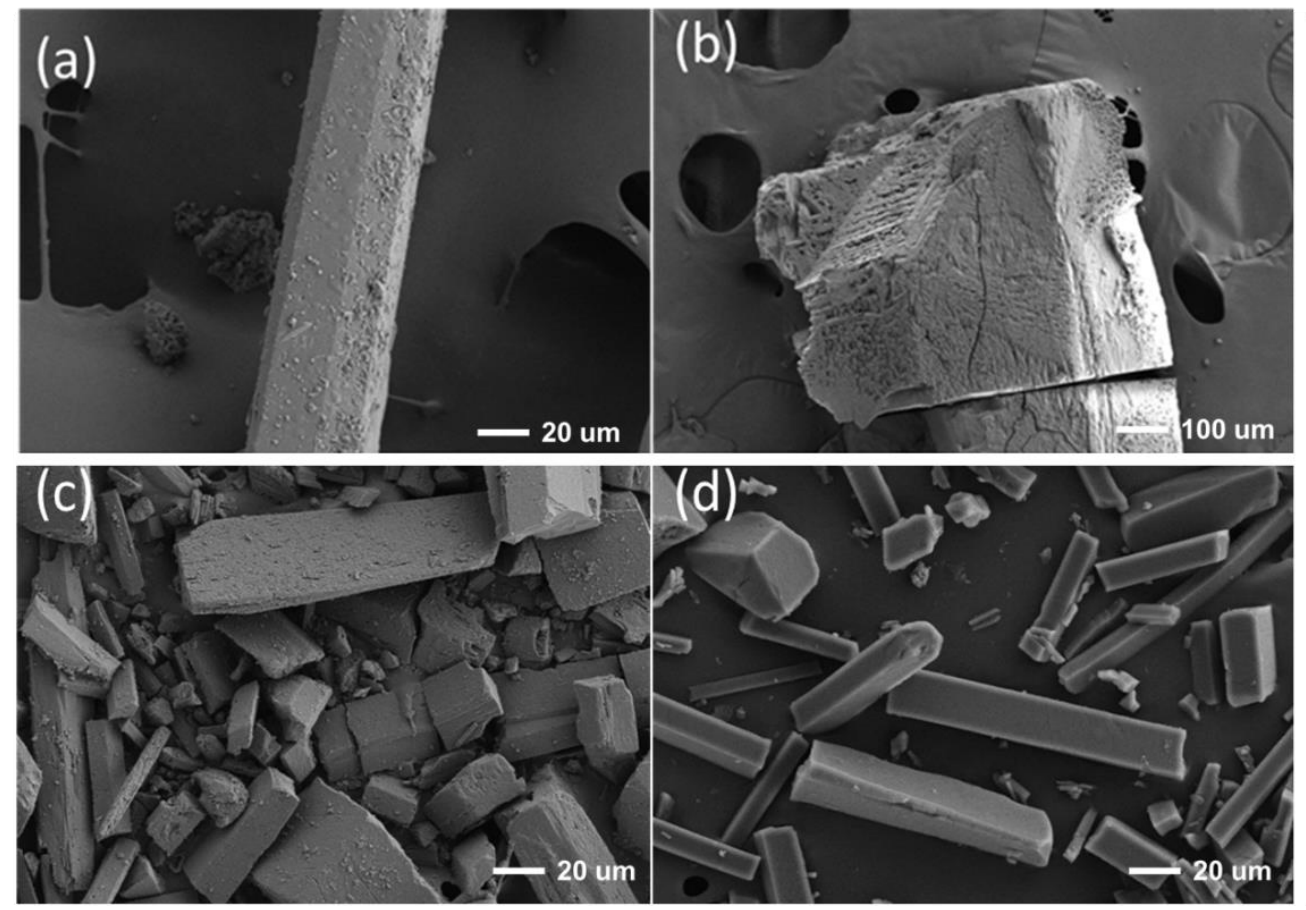

Figure S16. SEM images of the morphology and the digital images of iHOFs. iHOF-

1 (a); iHOF-2 (b); iHOF-3 (c) and iHOF-4(d).

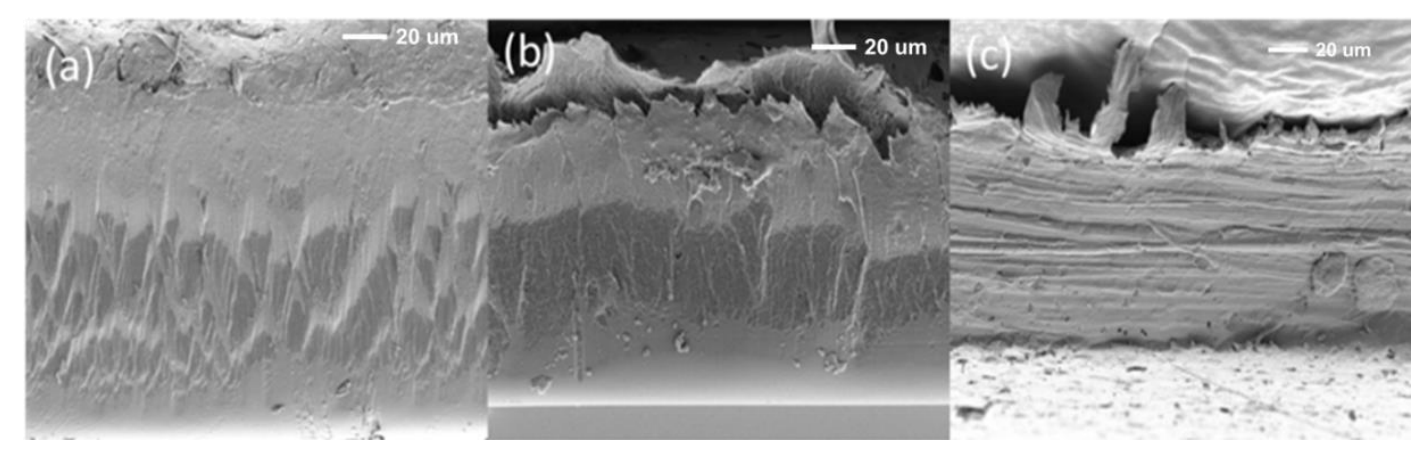

Figure S17. SEM images of the morphology of membrane. (a) The cross sections of recast Nafion; (b) The cross sections of 6\%-iHOF-3/Nafion composite membranes;(c) The the cross sections of $6 \%$-iHOF-4/Nafion composite membranes. 
Section S7. Atomic force microscopy.

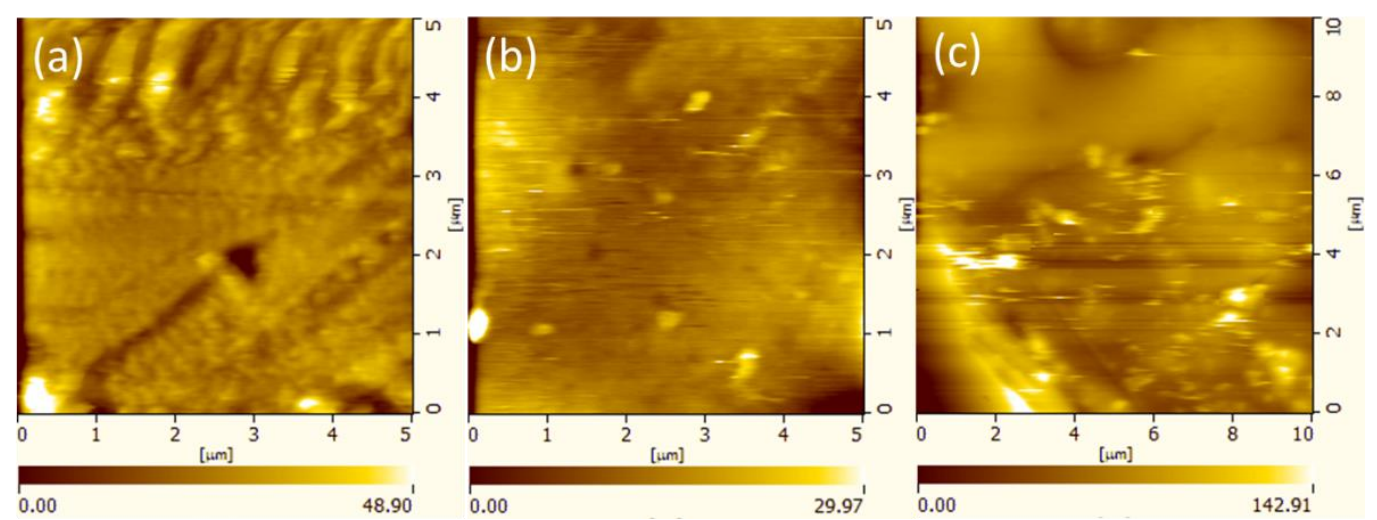

Figure S18. Atomic force microscopy date of composite membranes. Atomic force microscopy date of recast Nafion (a); 6\%-iHOF-3/Nafion composite membranes (b); 6\%-iHOF-3/Nafion composite membranes (c). 
Section S8. Thermogravimetric analysis.

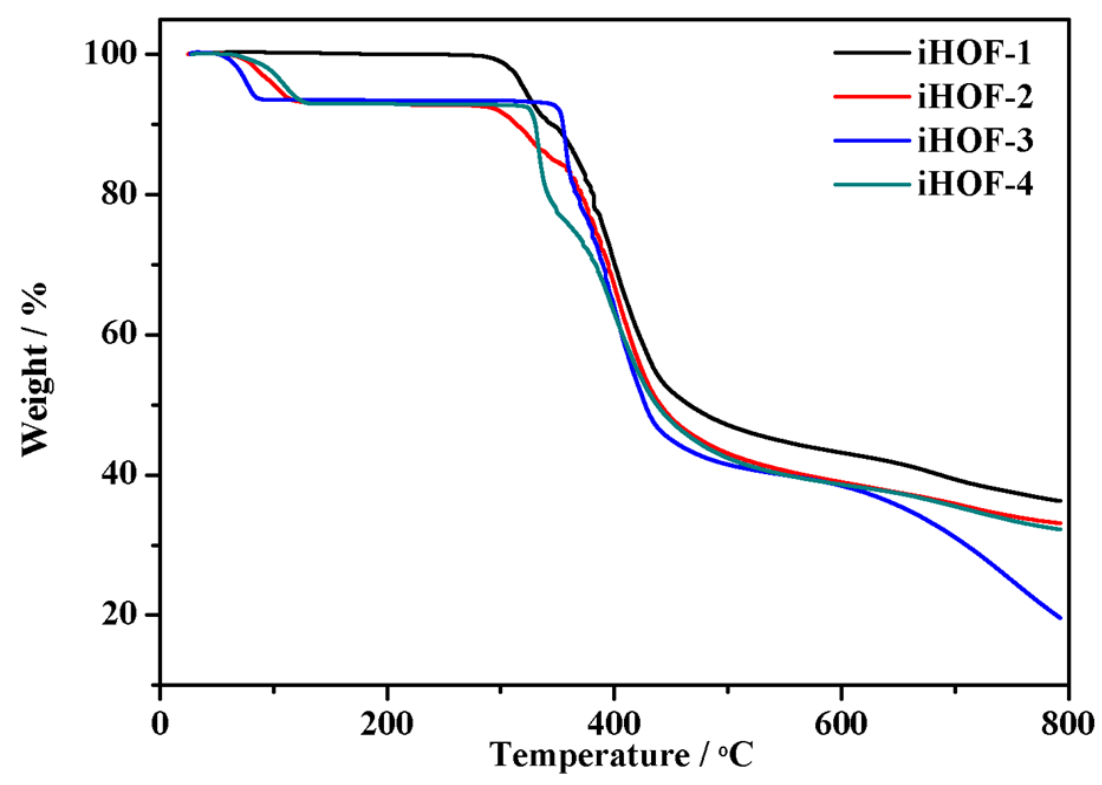

Figure S19. TGA plots of iHOFs 1-4. 


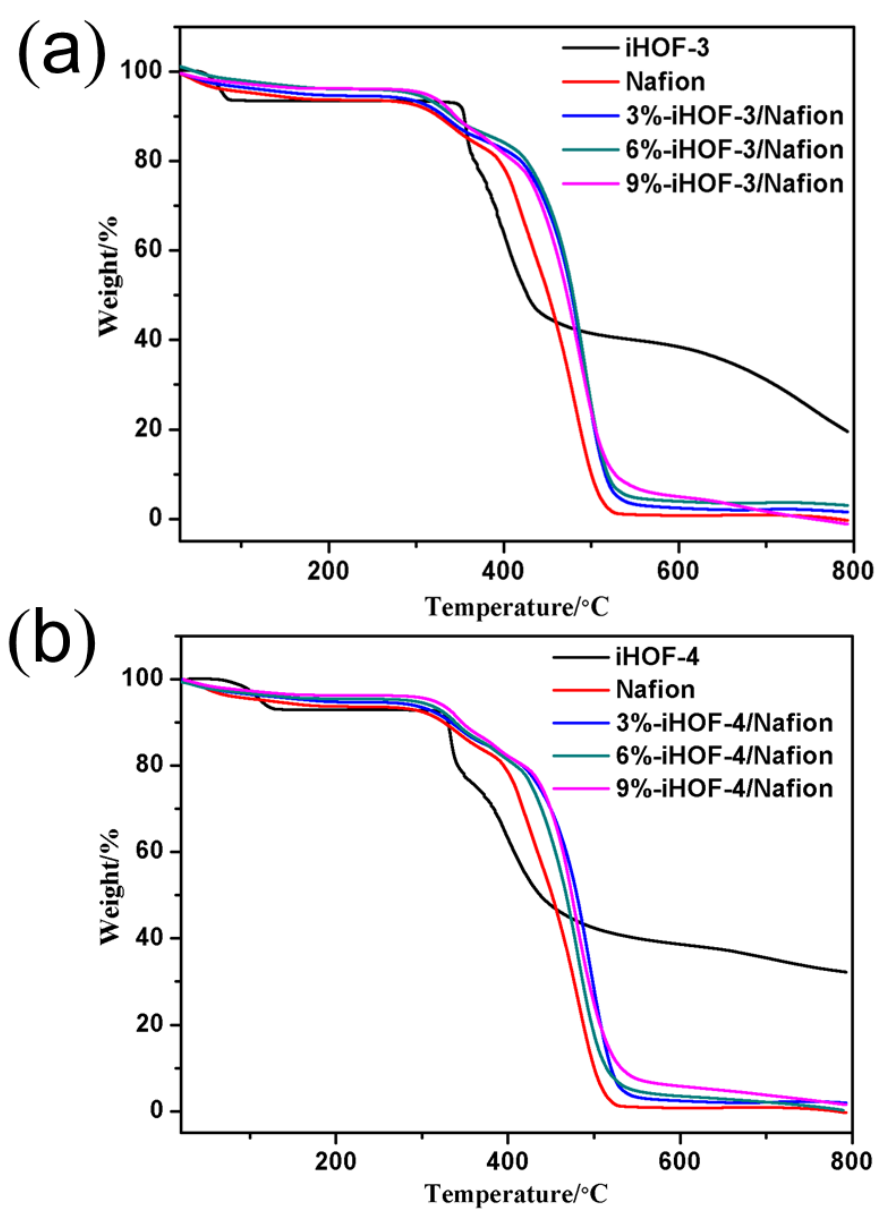

Figure S20. TGA plots of Nafion/iHOF composite membranes (a) TG curves of the

Nafion/iHOF-3 membranes; (b) TG curves of the Nafion/iHOF-4 membranes. 
Section S9. Proton conductivity.

(a)

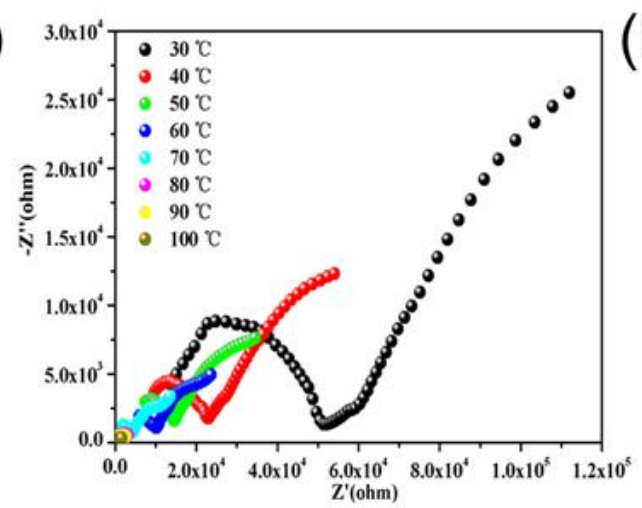

(c)

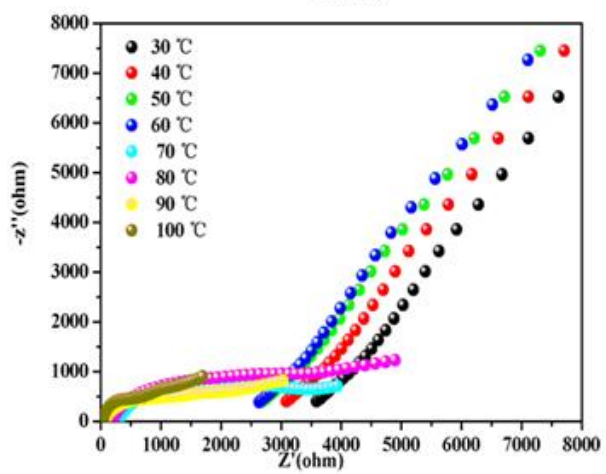

(e)

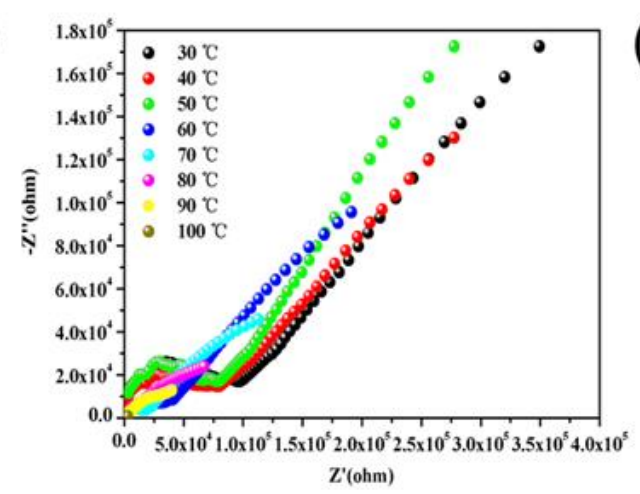

(b)

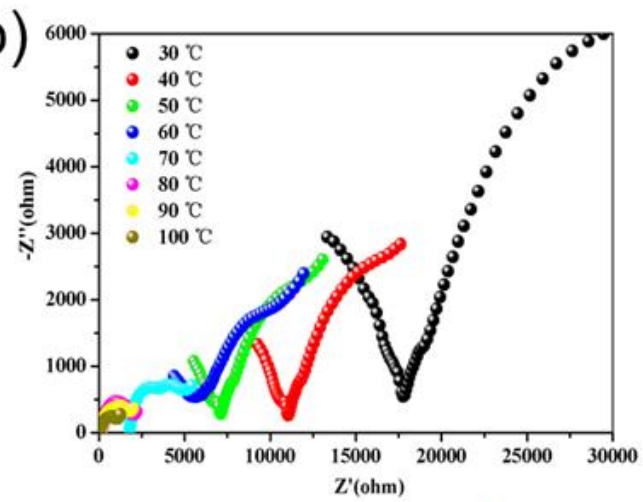

(d)

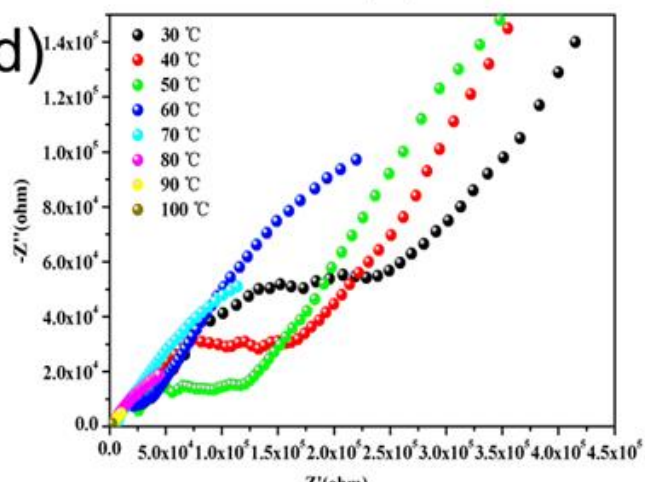
$Z^{\prime}(\mathrm{ohm})$

(f)

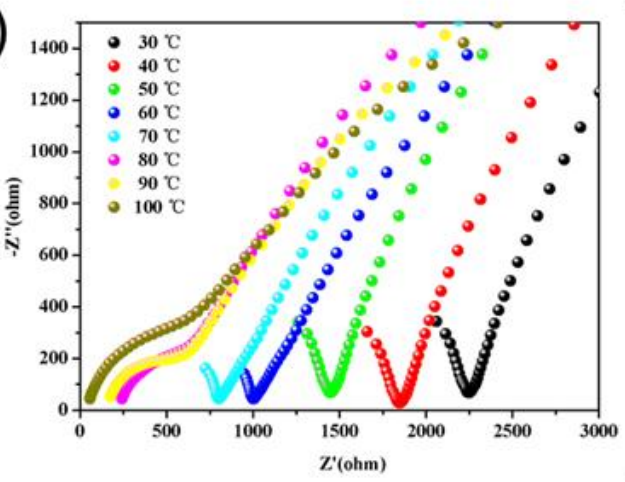

Figure S21. Impedance spectra of iHOF-3 and iHOF-4: (a) Nyquist plots of iHOF-3 at 75\% RH; (b) Nyquist plots of iHOF-3 at 85\% RH; (c) Nyquist plots of iHOF-3 at 93\% RH; (d) Nyquist plots of iHOF-4 at 75\% RH; (e) Nyquist plots of iHOF-4 at 85\% RH; (f) Nyquist plots of iHOF-4 at 93\% RH. 

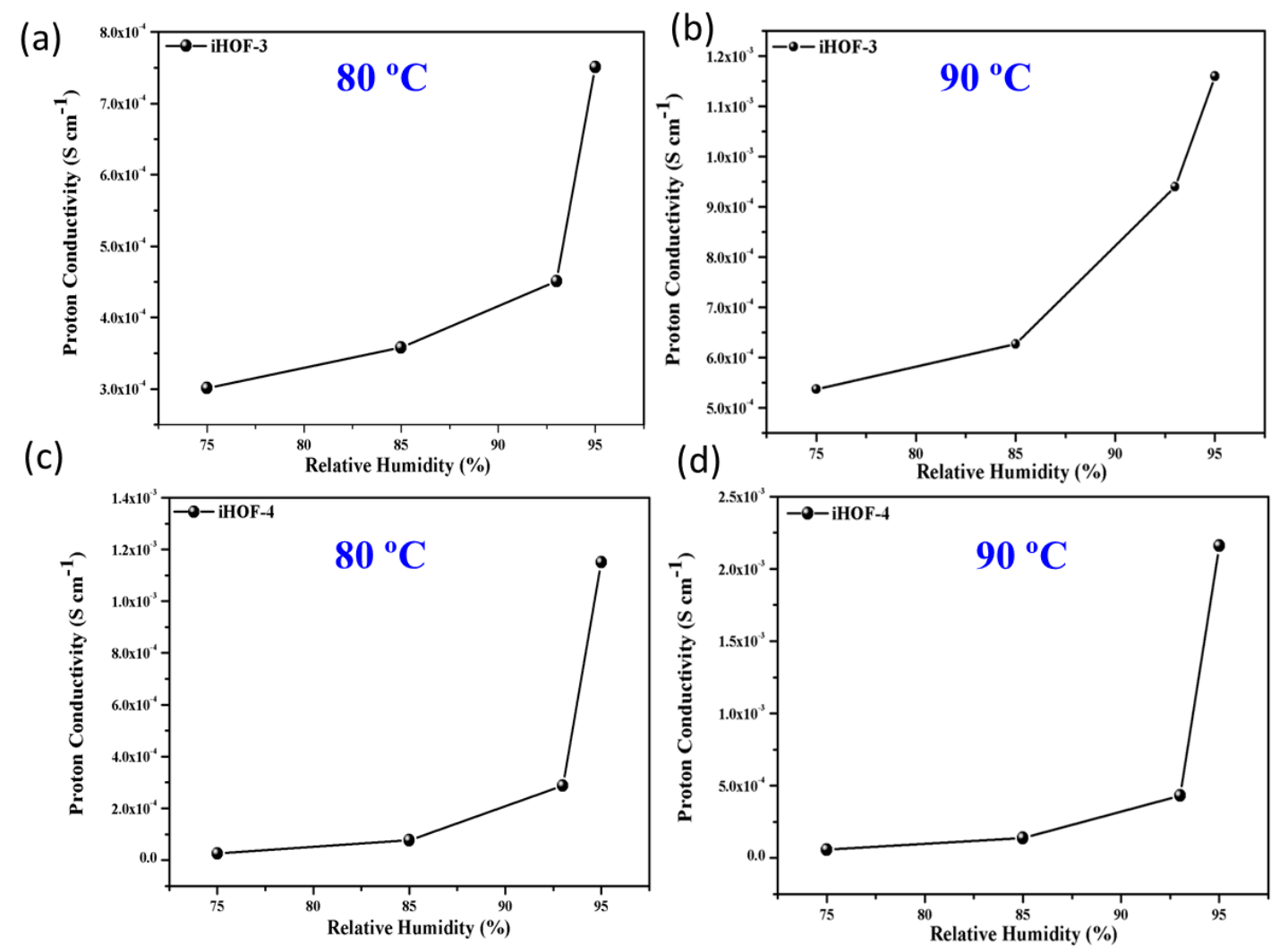

Figure S22. Relative-Humidity proton conductivity of iHOF-3 and iHOF-4 at $80{ }^{\circ} \mathrm{C}$ and $90{ }^{\circ} \mathrm{C}$. 
Table S3. Proton conductivities $\left(\mathrm{S} \cdot \mathrm{cm}^{-1}\right)$ for iHOF-3 at $30 \sim 100{ }^{\circ} \mathrm{C}$ and different Relative humidity (RH).

\begin{tabular}{ccccccccc}
\hline & $30{ }^{\circ} \mathrm{C}$ & $40{ }^{\circ} \mathrm{C}$ & $50{ }^{\circ} \mathrm{C}$ & $60{ }^{\circ} \mathrm{C}$ & $70{ }^{\circ} \mathrm{C}$ & $80{ }^{\circ} \mathrm{C}$ & $90{ }^{\circ} \mathrm{C}$ & $100{ }^{\circ} \mathrm{C}$ \\
\hline $75 \%$ & $1.50 \times 10^{-6}$ & $3.31 \times 10^{-6}$ & $5.01 \times 10^{-6}$ & $7.51 \times 10^{-6}$ & $1.07 \times 10^{-5}$ & $3.01 \times 10^{-4}$ & $5.37 \times 10^{-4}$ & $1.07 \times 10^{-3}$ \\
& & & & & & & & \\
$85 \%$ & $5.37 \times 10^{-6}$ & $9.40 \times 10^{-6}$ & $1.25 \times 10^{-5}$ & $1.88 \times 10^{-5}$ & $4.18 \times 10^{-5}$ & $4.18 \times 10^{-4}$ & $6.27 \times 10^{-4}$ & $1.37 \times 10^{-3}$ \\
& & & & & & & & \\
$93 \%$ & $2.15 \times 10^{-5}$ & $2.51 \times 10^{-5}$ & $2.78 \times 10^{-5}$ & $3.01 \times 10^{-5}$ & $2.43 \times 10^{-4}$ & $3.58 \times 10^{-4}$ & $9.04 \times 10^{-4}$ & $1.88 \times 10^{-3}$ \\
& & & & & & & & \\
$98 \%$ & $3.13 \times 10^{-5}$ & $4.18 \times 10^{-5}$ & $5.37 \times 10^{-4}$ & $3.42 \times 10^{-4}$ & $5.01 \times 10^{-4}$ & $7.51 \times 10^{-4}$ & $1.16 \times 10^{-3}$ & $1.67 \times 10^{-3}$ \\
\hline
\end{tabular}

Table S4. Proton conductivities $\left(\mathrm{S} \cdot \mathrm{cm}^{-1}\right)$ for iHOF-4 at $30 \sim 100{ }^{\circ} \mathrm{C}$ and different Relative humidity (RH).

\begin{tabular}{ccccccccc}
\hline & $30{ }^{\circ} \mathrm{C}$ & $40{ }^{\circ} \mathrm{C}$ & $50{ }^{\circ} \mathrm{C}$ & $60{ }^{\circ} \mathrm{C}$ & $70{ }^{\circ} \mathrm{C}$ & $80{ }^{\circ} \mathrm{C}$ & $90{ }^{\circ} \mathrm{C}$ & $100{ }^{\circ} \mathrm{C}$ \\
\hline $75 \%$ & $2.30 \times 10^{-7}$ & $3.29 \times 10^{-7}$ & $5.76 \times 10^{-7}$ & $1.73 \times 10^{-6}$ & $1.15 \times 10^{-5}$ & $2.56 \times 10^{-5}$ & $5.76 \times 10^{-5}$ & $2.87 \times 10^{-4}$ \\
& & & & & & & & \\
$85 \%$ & $6.90 \times 10^{-7}$ & $8.63 \times 10^{-7}$ & $9.86 \times 10^{-7}$ & $1.72 \times 10^{-6}$ & $3.45 \times 10^{-5}$ & $7.67 \times 10^{-5}$ & $1.38 \times 10^{-4}$ & $4.32 \times 10^{-4}$ \\
& & & & & & & & \\
$93 \%$ & $3.45 \times 10^{-5}$ & $4.93 \times 10^{-5}$ & $5.76 \times 10^{-5}$ & $8.63 \times 10^{-5}$ & $1.15 \times 10^{-4}$ & $2.88 \times 10^{-4}$ & $4.31 \times 10^{-4}$ & $1.73 \times 10^{-3}$ \\
& & & & & & & & \\
$98 \%$ & $1.97 \times 10^{-4}$ & $2.38 \times 10^{-4}$ & $2.89 \times 10^{-4}$ & $4.31 \times 10^{-4}$ & $7.67 \times 10^{-4}$ & $1.15 \times 10^{-3}$ & $2.16 \times 10^{-3}$ & $2.76 \times 10^{-3}$ \\
\hline
\end{tabular}


(a)

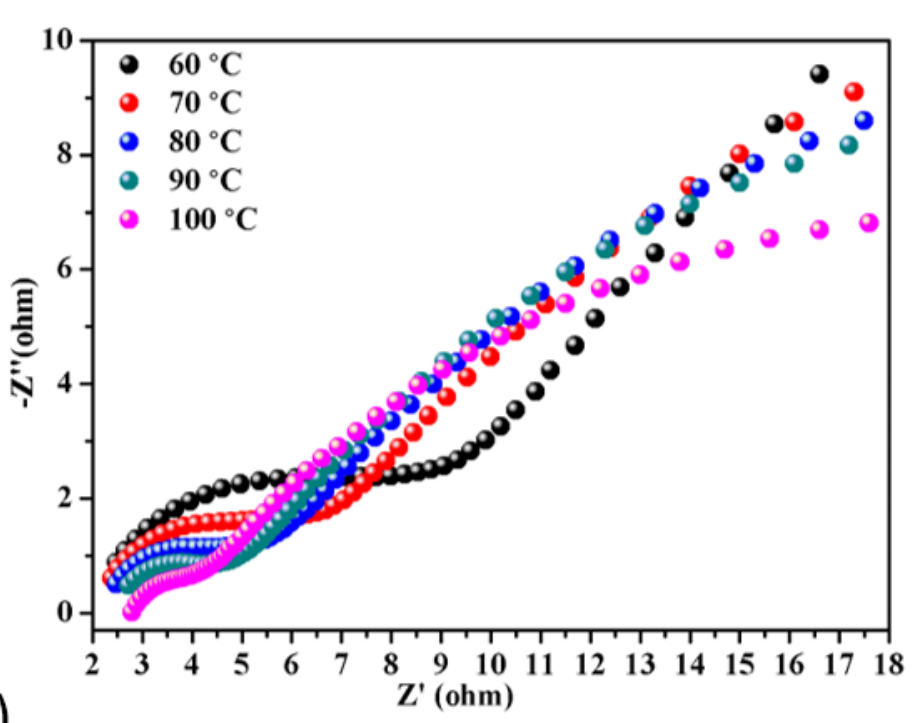

(b)

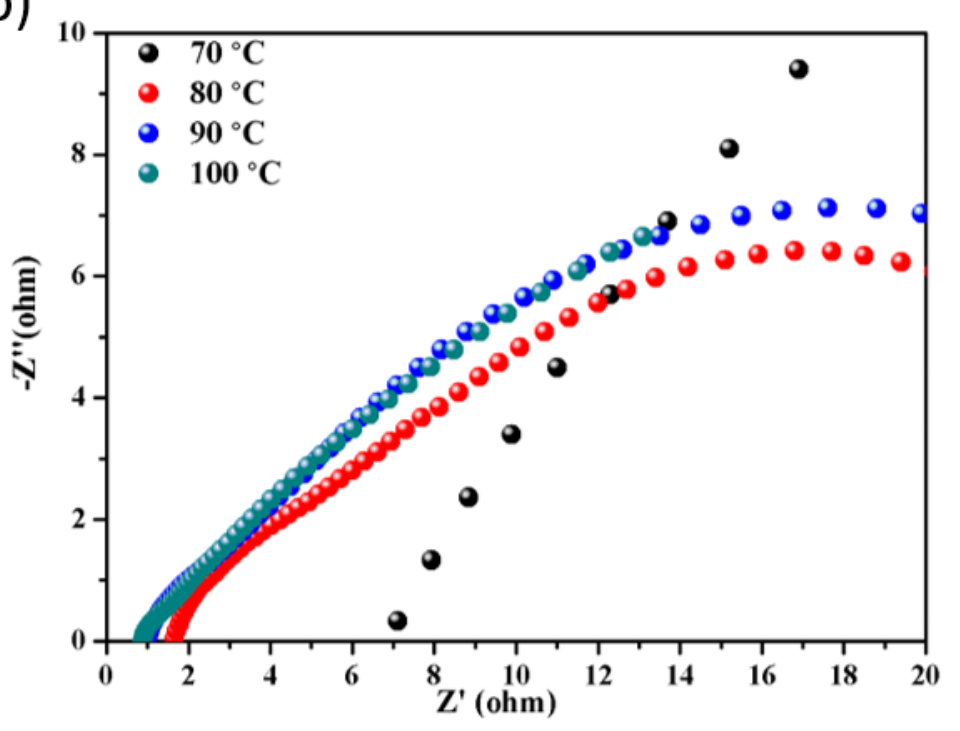

Figure S23. Temperature-dependent proton conductivity of 3\%-iHOF-3/Nafion (a) and 9\%-iHOF-3/Nafion (b) membranes at 98\% RH. 
(a)

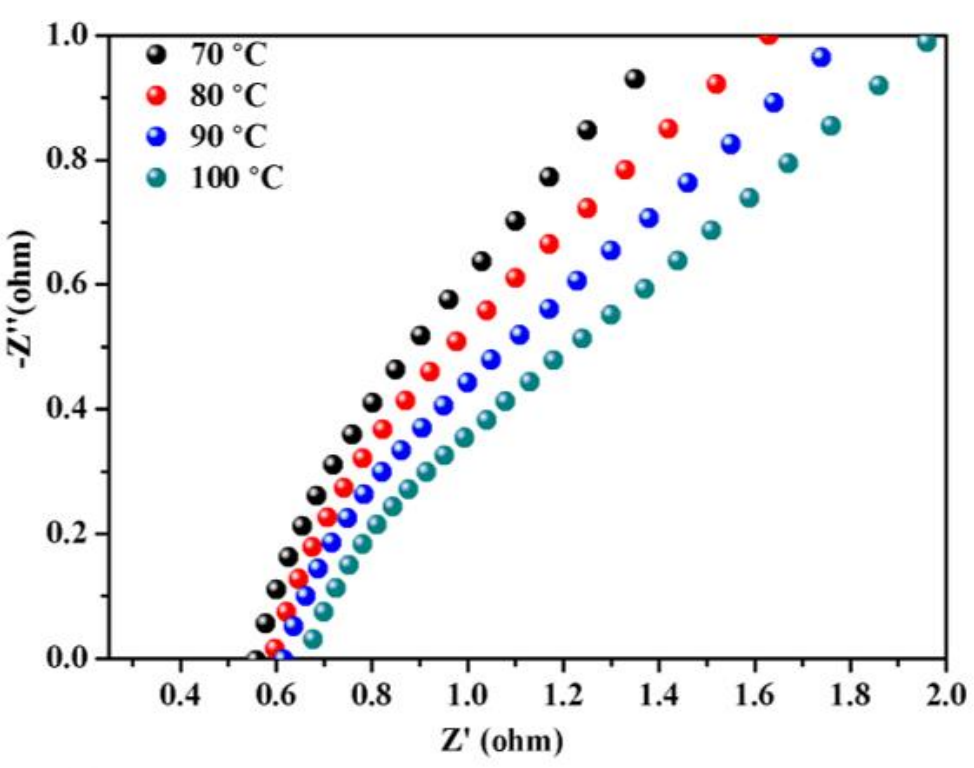

(b)

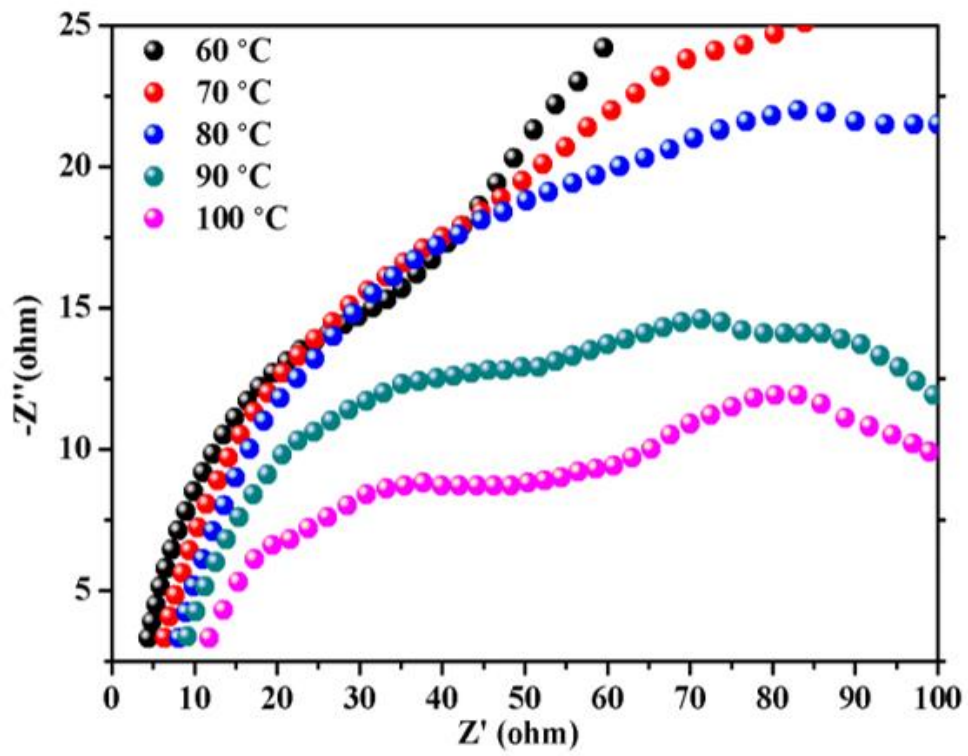

Figure S24. Temperature-dependent proton conductivity of 3\%-iHOF-4/Nafion (a) and 9\%-iHOF-4/Nafion (b) membranes at 98\% RH. 
Table S5. Comparison of proton conductivity and the maximum power density applied to PEMFCs of Nafion/iHOFs hybrid membranes with the reported Nafion-based membranes.

\begin{tabular}{|c|c|c|c|c|}
\hline $\begin{array}{l}\text { membrane } \\
\text { materials }\end{array}$ & $\begin{array}{l}\text { proton } \\
\text { conductivity } \\
\left(\mathbf{S} \cdot \mathbf{c m}^{-1}\right)\end{array}$ & $\begin{array}{l}\text { the maximum } \\
\text { power density } \\
\left(\mathrm{mW} \cdot \mathrm{cm}^{-2}\right)\end{array}$ & $\begin{array}{l}\text { temperature } \\
\text { and humidity } \\
\text { condition }\end{array}$ & references \\
\hline N/Tb-II & $3.61 \times 10^{-2}$ & 1138 & $\begin{array}{l}80^{\circ} \mathrm{C} \text { and } \\
100 \% \mathrm{RH}\end{array}$ & S7 \\
\hline N/SD-Eu-I & $3.44 \times 10^{-2}$ & 1075 & $\begin{array}{l}80^{\circ} \mathrm{C} \text { and } \\
100 \% \mathrm{RH}\end{array}$ & S7 \\
\hline Nafion/SmH ${ }_{2} \mathrm{SP}-3$ & $2.76 \times 10^{-2}$ & 946 & $\begin{array}{l}80^{\circ} \mathrm{C} \text { and } \\
100 \% \mathrm{RH}\end{array}$ & S8 \\
\hline Nafion/SmH ${ }_{2} \mathrm{SP}-5$ & $2.61 \times 10^{-2}$ & $\sim 800$ & $\begin{array}{l}80^{\circ} \mathrm{C} \text { and } \\
100 \% \mathrm{RH}\end{array}$ & S8 \\
\hline Nafion 211 & $8.3 \times 10^{-2}$ & 450 & $45^{\circ} \mathrm{C}$ & S9 \\
\hline iHOF-4 & $4.98 \times 10^{-3}$ & 420 & $\begin{array}{l}80^{\circ} \mathrm{C} \text { and } \\
100 \% \mathrm{RH}\end{array}$ & This work \\
\hline rN212/T1.5 & $\sim 1.3 \times 10^{-2}$ & 420 & l & S10 \\
\hline Nafion 212 & $8.3 \times 10^{-2}$ & 400 & $45^{\circ} \mathrm{C}$ & S9 \\
\hline iHOF-3 & $1.13 \times 10^{-2}$ & 360 & $\begin{array}{l}80^{\circ} \mathrm{C} \text { and } \\
100 \% \mathrm{RH}\end{array}$ & This work \\
\hline $\begin{array}{l}\text { Nafion/H3 } \mathrm{HO}_{4} @ \text { S1- } \\
15\end{array}$ & 0.137 & 277.8 & $60^{\circ} \mathrm{C}$ & S11 \\
\hline $\begin{array}{l}\text { Zn-MOF- } \\
\text { NH }_{3} / \text { Nafion-5 }\end{array}$ & $2.13 \times 10^{-2}$ & 212 & $60^{\circ} \mathrm{C}$ & S12 \\
\hline Nafion/Z-COF-10 & 0.22 & $\sim 170$ & $\begin{array}{l}80^{\circ} \mathrm{C} \text { and } \\
100 \% \mathrm{RH}\end{array}$ & S13 \\
\hline
\end{tabular}


Section S10. Stress-strain curves.
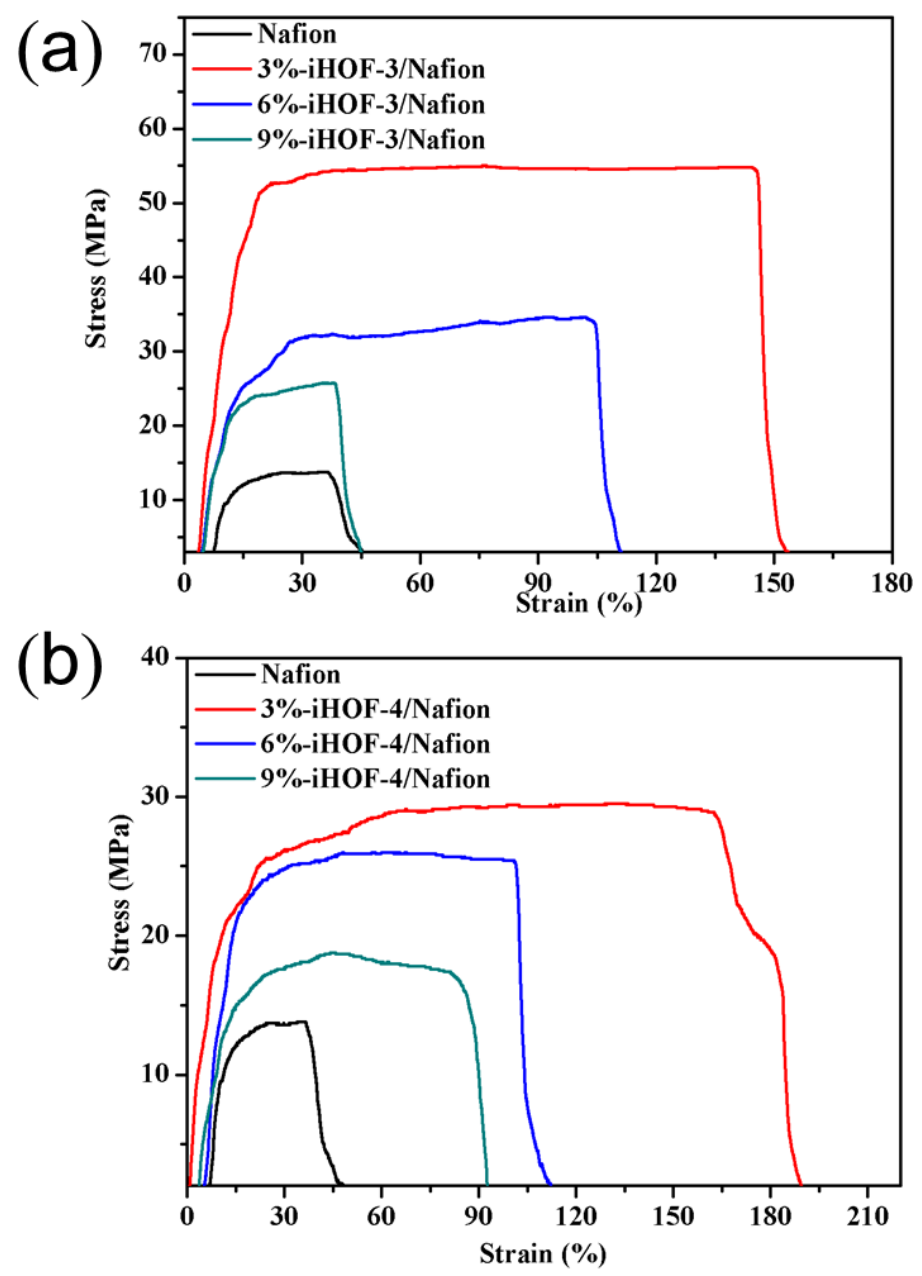

Figure S25. Stress-strain curves of the membranes at room temperature. 


\section{Section S11. References.}

[S1] Downes, J. E. J. Chem. SOC. 1967, 2192.

[S2] SMART and SAINT. Area Detector Control and Integration Software; Siemens Analytical X-Ray Systems, Inc.: Madison, W. I., 1996.

[S3] Sheldrick, G. M. SADABS: Program for Empirical Absorption Correction of Area Detector Data, University of Göttingen, Germany, 1996.

[S4] Sheldrick, G. M. Acta Cryst. A, 2008, 64, 112.

[S5] Dolomanov, O. V.; Bourhis, L. J.; Gildea, R. J.; Howard, J. A. K.; Puschmann, H. J. Appl. Cryst., 2009, 42, 339.

[S6] Sheldrick, G. M. Acta Cryst. C, 2015, 71, 3.

[S7] Salcedo, I. R.; Colodrero, R. M. P.; Bazaga-García, M.; López-González, M.; del Río, C.; Xanthopoulos, K.; Demadis, K. D.; Hix, G. B.; Furasova, A. D.; Choquesillo-Lazarte, D.; Olivera-Pastor, P.; Cabeza, A. Phase Transformation Dynamics in Sulfate-Loaded Lanthanide Triphosphonates. Proton Conductivity and Application as Fillers in PEMFCs. ACS Appl. Mater. Interfaces 2021, 13, 15279 .

[S8] Bazaga-García, M.; Salcedo, I. R.; Colodrero, R. M. P.; Xanthopoulos, K.; Villemin, D.; Stock, N.; López-González, M.; del Río, C.; Losilla, E. R.; Cabeza, A.; Demadis, K. D.; Olivera-Pastor, P. Layered Lanthanide Sulfophosphonates and Their Proton Conduction Properties in Membrane Electrode Assemblies. Chem. Mater. 2019, 31, 9625 .

[S9] Liu, W.; Xie, Y.; Liu, J.; Jie, X.; Gu, J.; Zou, Z. Experimental Study of Proton Exchange Membrane Fuel Cells Using Nafion 212 and Nafion 211 for Portable Application at Ambient Pressure and Temperature Conditions. Int. J. Hydrogen Energy 2012, 37, 6473.

[S10] Ye, J.; Zhao, X.; Ma, Y.; Su, J.; Xiang, C.; Zhao, K.; Ding, M.; Jia, C.; Sun, L. Hybrid Membranes Dispersed with Superhydrophilic $\mathrm{TiO}_{2}$ Nanotubes Toward Ultra-Stable and High-Performance Vanadium Redox Flow Batteries. Adv. Energy Mater. 2020, 1904041. 
[S11] Yin, Y.; Li, Z.; Yang, X.; Cao, L.; Wang, C.; Zhang, B.; Wu, H.; Jiang, Z. Enhanced Proton Conductivity of Nafion Composite Membrane by Incorporating Phosphoric Acid-Loaded Covalent Organic Framework. J. Power Sources 2016, $332,265$.

[S12] Wang, H.; Zhao, Y.; Shao, Z.; Xu, W.; Wu, Q.; Ding, X.; Hou, H. Proton Conduction of Nafion Hybrid Membranes Promoted by $\mathrm{NH}_{3}$-Modified Zn-MOF with Host-Guest Collaborative Hydrogen Bonds for $\mathrm{H}_{2} / \mathrm{O}_{2}$ Fuel Cell Applications. ACS Appl. Mater. Interfaces 2021, 13, 7485.

[S13] Li, Y.; Wu, H.; Yin, Y.; Cao, L.; He, X.; Shi, B.; Li, J.; Xu, M.; Jiang, Z.; Fabrication of Nafion/Zwitterion-Functionalized Covalent Organic Framework Composite Membranes with Improved Proton Conductivity. J. Membr. Sci. 2018, 568,1 . 